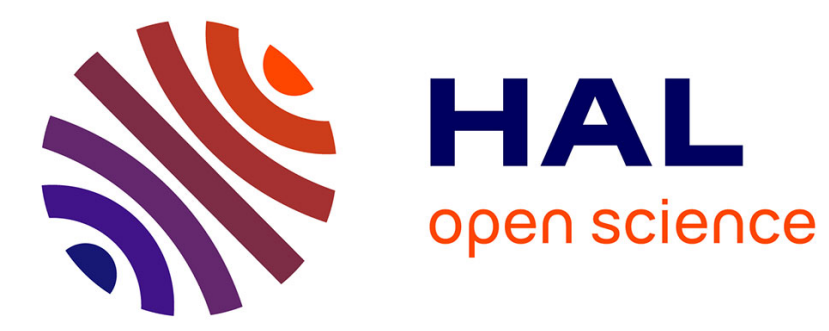

\title{
Hyperfine Transitions in the first Overtone mode of Hydrogen Deuteride
}

\author{
Patrick Dupre
}

\section{To cite this version:}

Patrick Dupre. Hyperfine Transitions in the first Overtone mode of Hydrogen Deuteride. Physical Review A, 2020. hal-03080222

\section{HAL Id: hal-03080222 \\ https://u-bourgogne.hal.science/hal-03080222}

Submitted on 17 Dec 2020

HAL is a multi-disciplinary open access archive for the deposit and dissemination of scientific research documents, whether they are published or not. The documents may come from teaching and research institutions in France or abroad, or from public or private research centers.
L'archive ouverte pluridisciplinaire HAL, est destinée au dépôt et à la diffusion de documents scientifiques de niveau recherche, publiés ou non, émanant des établissements d'enseignement et de recherche français ou étrangers, des laboratoires publics ou privés. 


\title{
Hyperfine Transitions in the first Overtone mode of Hydrogen Deuteride
}

\author{
Patrick Duprê $\hat{}^{*}$ \\ Laboratoire Interdisciplinaire Carnot de Bourgogne, UMR CNRS 6303,
}

9 Avenue Alain Savary, BP 47870, 21078 Dijon Cedex, France

(Dated: December 17, 2020)

Beyond the metrology and computational challenges associated with molecular hydrogen, key data are expected to assess the physics of simple molecular systems, and even the New Physics beyond the Standard Model. To assist the deciphering of Doppler-free spectra obtained at very high accuracy $\left(\sim 10^{-9}\right)$, we report on hyperfine transitions of HD in the lowest vibrational levels of the ground electronic state. Using the spin-rotation, nuclear spin-spin and quadrupolar hyperfine couplings determined by means of high-level quantum-chemical calculations, the hyperfine energy levels, and the associated line intensity have been obtained by using tensorial momentum algebra. To illustrate our purpose, the hyperfine line intensity of two specific transitions $\left(P_{1}\right.$ and $\left.R_{1}\right)$ belonging to the first overtone of HD is reported and commented on. The calculated stick spectra emphasize the experimental challenge (in terms of sensitivity and of spectral resolution) associated with the spectral analysis, because the lines can be apart by less than $10 \mathrm{kHz}$.

PACS numbers: 31.15.aj, 33.15.Pw, 42.62.Eh

Keywords: Molecular hydrogen, Hyperfine Hamiltonian, Hyperfine electric transitions, Saturated Absorption, Tensorial algebra, Herman-Wallis coefficients 


\section{INTRODUCTION}

Since the pioneering observations of "forbidden" vibration-rotation transitions of HD (the most abundant isotopologues of the hydrogen molecule) by Herzberg in the 1950s [1, 2], the complete interpretation of the intensity of these lines has generated a lot of discussions in the literature, even questioning the role of the nonadiabatic couplings in the framework of the Born-Oppenheimer approximation (BOA) [3] as well as the collisional intermolecular potential. Actually, this BOA framework remains pivotal, because of the tractability of the computational solutions [4]. Solving the Schrödinger Equation beyond the BOA, i.e., without considering the associated corrections [5, and even including relativistic effects [6], is nowadays possible. Remarkable computational progresses have been attained on the hydrogen isotopologues thanks to the nonadiabatic perturbation theory (NAPT) or to a modified nonadiabatic approach[7]. The relativistic effects can be in principle considered [8, 9] as well as the QED theory [10]. Restringing the discussion to the hydrogen isotopologue HD, it is worth noting that only the calculation of the transition frequencies has benefited of the last computational developments, either by a variational approach [11-13], or by NAPT[14], but not the vibrationrotation line intensities. The best estimated accuracy on the calculated vibration-rotation line position reaches $1 \mathrm{MHz}$. Nevertheless, the internal hyperfine couplings (typically for HD, HT, and DT) have just been ignored, except recently, with calculation of the nuclear spin-spin scalar coupling[15]. Accounting for these contributions is crucial to challenge the sub-MHz accuracy which is targeted by very recent experiments.

The recent implementations of very high-resolution spectroscopy setups reveal new controverted behaviors on HD [16, 17]. Then, there are new requirements for examining the internal structure (i.e., beyond the usual rotational structure) of molecules even in the Near-Infrared (NIR) domain, while the study of these structures has been regularly the private garden of microwave spectroscopy. Laser spectroscopy with sub-Doppler resolution (like saturated absorption) can be employed to interrogate the hyperfine structure of molecules at the highest precision (including the microwave range). Indeed, spectroscopy data at the metrology level can now be obtained in different frequency ranges thanks to the assistance of the Optical Frequency Combs (OFC) providing an incredible spectral precision[18]. From a strict metrology point of view, "pure" or structureless transitions (like those of para- $\mathrm{H}_{2}$ ) must be in priority targeted to facilitate the data analysis. Unfortunately, such experimental data are not available yet. Nevertheless, data on akin systems are already available, like on HD in the NIR range 16, 17]: they ask for a challenging analysis when a spectral accuracy of $\sim 10^{-9}$ is demonstrated.

Molecular hydrogen is the molecule to choose when challenging new determination of the proton-to-electron mass ratio for example, and ultimately when considering the Physics beyond the Standard Model. Currently, the rovibrational transitions of molecular hydrogen offer the unique advantage to be ab initio tractable from the fundamental principles with a level of accuracy approaching the best experimental resolution 17 19. However, while in para- $\mathrm{H}_{2}\left(\right.$ and para- $\mathrm{T}_{2}$ ), $I=0$ transitions are free of hyperfine complexity, all the other isotopologues (including ortho- $\mathrm{H}_{2}$ and $-\mathrm{T}_{2}$ ) exhibit an internal structure due to the nonzero total nuclear spin. The resulting contribution of these hyperfine couplings to the shape of the rotational transitions have only been partially considered nowadays, despite these couplings probably complexifying the resonance shapes observable under nonlinear spectroscopy, cf. by inducing crossover resonances. Furthermore, the hyperfine structure of the $I \neq 0$ isotopologues potentially exhibits a (weak) sensitivity to the proton-to-electron mass ratio which may have to be accurately investigated. In addition, hyperfine interactions are 
of crucial importance if they accidentally mix ortho and para energy levels in molecules exhibiting a $u / g$ symmetry, these interactions can reveal "forbidden" transitions, and give rise to nuclear spin conversion [20].

In closed shell molecules, the hyperfine structure when it exists (it requires for nuclei with spin magnetic moments and/or with electric quadrupole moments), remains "microscopic", while in open shell molecules the hyperfine couplings cannot be ignored, usually. $\mathrm{NO}_{2}$ is a good example for an open shell molecule, given what can be observed in the NIR-visible range under saturated absorption 21. Another example is the ion $\mathrm{HD}^{+}$which exhibits very large hyperfine coupling intensities compared with those of $\mathrm{HD}[22$. Indeed, the scheme of energy level coupling by an external electromagnetic field ( $V, \Lambda$, or $N$ configuration) illustrates the additional complexity linked to the interference between electrical dipolar transitions, arising crossover resonances resulting from nonlinear couplings in a counterpropagating beam geometry, i.e., beyond the linear absorption under weak electromagnetic fields. Crossover resonances appear when, at least, two transitions share the same energy level, and when the transition frequency difference is less than the thermal Doppler broadening (or of the same order). Literally, a single molecule absorbs two counter-propagating photons if the Doppler shift allows exactly compensation of the energy difference between the levels coupled by the external electromagnetic field. Indeed, nonlinear resonances, even when they are associated with weak transitions, remain perfect probes for investigating closed-shell molecules exhibiting hyperfine structures. Thus we anticipate that the present work should provide the key inputs for deciphering the resonance shapes observed on HD as previously mentioned.

Although the inversion symmetry of the full molecular Hamiltonian for homonuclear diatomic molecules (i.e., $\mathrm{X}_{2}$, where $\mathrm{X}=\mathrm{H}, \mathrm{D}$, etc..) makes these species rigorously nonpolar, a small permanent electric dipole moment exists in the corresponding heteroisotopic isotopologues (like HD), thereby making vibrational dipolar transitions of these molecules weakly allowed. While it is perhaps traditional to associate this weak electrical charge asymmetry (the density of charge located on the deuteron differs slightly from that located on the proton) with "nonadiabatic effects" that mix different (clamped-nucleus) adiabatic wave functions 23 , 30 , recent works, like those based on the so called Post-BO approach $7,31,33$, on the so-called non-BO variational approach 34 35, or on the approximate adiabatic variational approach [36] have convincingly demonstrated that the overwhelming majority of this effect resides in the so-called adiabatic (diagonal Born-Oppenheimer) correction. Hence within this perspective, the intensity of fundamental and overtone vibrational transitions in molecules like HD can be calculated by the usual methods as currently done for the calculation of the hyperfine couplings.

Beyond the weak electrical dipolar transitions mentioned above to probe HD, there are few experimental alternatives. Nevertheless, the existence of electric quadrupolar moments 37 should provide complementary inputs as soon as the saturated absorption of these transitions will have been demonstrated.

The collisional dynamic of molecular hydrogen is dominated by strong velocity-changing collision (VCC) giving rise to the well-established Dicke effect, or "motional" narrowing of the Doppler profile of the transitions in the "high" pressure range, providing non-Voigt profiles [38, 39]. Actually, the detailed analysis of the speed-dependence line broadening and frequency shift (under linear absorption) remains a challenge. Investigating these profiles under saturation conditions may even seem to be a more complex challenge. Despite these difficulties, understanding the Lamb-dip behavior under saturation regimes may appear as an opportunity for probing the HD intermolecular potential. Preliminary data are 
now available and their analysis requires full attention, particularly, by extensively investigating the pressure and power dependencies. At low pressures, the molecular interaction transit time, resulting from the finite size of the excitation beam is undoubtedly a source of additional complexity since resonance super-narrowing effects may be anticipated as it has been discussed on several molecular systems $40-44$. Actually, collisional speed-dependence frequency shift 45 may contribute to the asymmetric resonance profiles observed on HD.

The hyperfine structure of the ground vibrational state of HD has been known since the late 1950s, thanks to the nuclear radio-frequency spectroscopy work of Ramsey and collaborators carried out by applying a magnetic field on a molecular beam 46, 47]. Actually, at zero magnetic field, the hyperfine structure spreads over several hundreds of $\mathrm{kHz}$, depending on the transition considered. Nowadays, saturated absorption of weakly absorbing transitions can be observed in the NIR domain with a frequency precision much less than the spectral extension of the Doppler-free rovibrational transitions. Nevertheless, the current spectral resolution does not allow for unambiguously revealing the hyperfine structure. To accommodate disentangling the spectral complexity associated with the hyperfine structure of HD, we report here, i) succinctly on the hyperfine constants characterizing the spin-rotation, nuclear spin-spin dipolar and quadrupolar interactions of HD for $v=0-2$ (section IIA, ii) on the deduced hyperfine energy splittings which are validated versus Ramsey's data (sections IIF and II G, iii) on the Herman-Wallis coefficients of the overtone band $v: 2 \leftarrow 0$ (section III B, iv) on the hyperfine linear line intensity (in the electric dipole approximation) associated with the molecular absorption (section III C, and v) on the spectrum of two specific transitions ( $P_{1}$ and $\left.R_{1}\right)$ of the overtone considered (section III D. For these calculations, we intensively use the irreducible tensorial algebra following Brown and Carrington 48 .

The current calculations can be considered as a step forward in understanding the line shape of the HD resonances between the first vibrational modes, i.e., beyond the rotational structure. The algebraic approach presented in the following can be easily extended to other overtones of HD, and even to molecular systems exhibiting strong hyperfine similarities. This paper also corrects preliminary data previously displayed on the upper part of Fig. 2 of Ref. [16].

The paper is mainly divided in three parts: section II devoted to the calculation of the hyperfine levels, section III devoted to the calculation of the line intensity, followed by a general Discussion in section IV]

\section{HYPERFINE INTERACTIONS}

\section{A. Hyperfine Constants}

Quantum-chemical calculations [49] were carried out at the coupled-cluster single and double CCSD level[50], which is equivalent to a full configuration-interaction (FCI) treatment for a two-electron system such as HD. The basis set used was the uncontracted cc-pV6Z set[51] which consists of $10 s, 5 p, 4 d, 3 f, 2 g$, and $1 h$ functions for the hydrogen and deuterium. The harmonic force constant was obtained by using the second derivatives of the energy evaluated analytically[52]. In a second step, the vibrational corrections to all hyperfine constants have been computed by using a discrete variable representation (DVR) scheme [53] as described in Ref. [54]. The calculations were carried out at a very high level of theory in order to ensure a reliable simulation of the corresponding spectrum. However, there are remaining error sources such as basis-set truncation, neglect of the relativistic effects, non-Born-Oppenheimer 
contributions, and inaccuracies in the used potential curve. All together, they are estimated to be less than $0.5 \mathrm{kHz}$. All calculations have been performed with a local version of the CFOUR quantum-chemical package [55].

\section{B. Coupling Scheme}

In the absence of strong magnetic or electric field, the trivial coupling scheme to calculate the eigenenergies, is a coupled basis involving three angular momenta. However, having to deal with three angular momenta, none of the three possible coupling scheme $\left(\boldsymbol{N}+\boldsymbol{i}_{\mathrm{D}}, \boldsymbol{N}+\boldsymbol{i}_{\mathrm{H}}\right.$, or $\left.\boldsymbol{i}_{\mathrm{H}}+\boldsymbol{i}_{\mathrm{D}}\right)$ provides only diagonal matrix elements, or even a trivial hierarchy resulting from the coupling terms. Therefore, we will make the choice of the following angular momentum coupling (the Hund's cases a) and b) are equivalent in the absence of electronic spin[56]). We set

$$
\boldsymbol{F}=\boldsymbol{F}_{1}+\boldsymbol{i}_{\mathrm{H}}
$$

where $\boldsymbol{F}$ is the total angular momentum (invariant), $\boldsymbol{i}_{\mathrm{H}}$ is the angular momentum of the proton, and where $\boldsymbol{F}_{1}$ is defined by

$$
\boldsymbol{F}_{1}=\boldsymbol{N}+\boldsymbol{i}_{\mathrm{D}}
$$

where $\boldsymbol{i}_{\mathrm{D}}$ is the deuteron angular momentum, and where $\boldsymbol{N}$ is the usual rotational angular momentum of a rigid rotor.

Thus the total adiabatic molecular wave function is defined by $\left|\psi_{e v} \Lambda ; N i_{\mathrm{D}} F_{1} i_{\mathrm{H}} F m_{F}\right\rangle$ where $\Lambda$ is in the projection of the electronic angular momentum on the molecule-fixed frame. The electronic and vibrational wave functions $\left(\psi_{e v}\right)$ are assumed to be unmixed. $m_{F}$ is the projection of the total angular momentum in the laboratory-fixed frame, providing a $2 \boldsymbol{F}+1$ Zeeman degeneracy at zero magnetic or electric field.

\section{Hamiltonians}

The different interactions can be written down by using Cartesian coordinates in the laboratory-fixed frame, as follows (see Refs. [57, 58] for additional details).

\section{Nuclear Spin-Rotation Interactions}

The two nuclear spin momenta are independently coupled to the molecule rotation which creates a magnetic field at the position of the nuclei[59]. For a linear molecule each (second-order) spin-rotation tensor is reduced to a single term. Two couplings are provided by the two spin-rotation Hamiltonians proportioned by their respective factor:

$$
\mathcal{H}_{n s r_{\mathrm{H}}}=c_{\mathrm{H}} \boldsymbol{i}_{\mathrm{H}} \cdot \boldsymbol{N}
$$


and

$$
\mathcal{H}_{n s r_{\mathrm{D}}}=c_{\mathrm{D}} \boldsymbol{i}_{\mathrm{D}} \cdot \boldsymbol{N}
$$

where $c_{\mathrm{H}}$ and $c_{\mathrm{D}}$ are the two spin-rotation constants associated with the proton and with the deuteron, respectively. Indeed, these constants are expectation values calculated for the specific electronic-vibration wave function (actually, the perpendicular components to the interatomic axis of the respective tensors) [58].

Dipole-dipole interactions between two nuclear spin magnetic moments

In the ensuing analyses the scalar (or isotropic) spin-spin interaction

$$
\mathcal{H}_{\text {dip }_{0}}=c_{s} \boldsymbol{i}_{\mathrm{D}} \cdot \boldsymbol{i}_{\mathrm{H}}
$$

has been treated independently from the second order tensor spin-spin interaction derived from the Breit equation 60

$$
\mathcal{H}_{\text {dip }}=g_{\mathrm{H}} g_{\mathrm{D}} \frac{\mu_{0} \mu_{N}^{2}}{4 \pi}\left[\frac{\boldsymbol{i}_{\mathrm{H}} \cdot \boldsymbol{i}_{\mathrm{D}}}{r_{\mathrm{HD}}^{3}}-\frac{3\left(\boldsymbol{i}_{\mathrm{H}} \cdot r_{\mathrm{HD}}\right)\left(\boldsymbol{i}_{\mathrm{D}} \cdot r_{\mathrm{HD}}\right)}{r_{\mathrm{HD}}^{5}}\right] \text {, }
$$

where $\boldsymbol{r}_{\mathrm{HD}}$ is the internuclear distance, $g_{\mathrm{H}}$ and $g_{\mathrm{D}}$ are the proton and deuteron $g$ factors, respectively, and where $\mu_{N}$ is the nuclear magneton $\left(\mu_{0} \simeq 4 \pi \times 10^{-7} \mathrm{H} / \mathrm{m}\right.$ is the vacuum permeability) 61 .

\section{Electric Quadrupolar interaction}

Nuclei with spin magnetic moment $\boldsymbol{Q}$ larger than $1 / 2$ interact with the gradient of the static electrical field $\nabla E$, following Townes and Schawlow 62

$$
\mathcal{H}_{\text {quad }}=-\frac{1}{6} \boldsymbol{Q}: \nabla E
$$

where “: ” represents a double inner product between two second-order tensors.

\section{Matrix Elements}

The matrix elements will be calculated by using the irreducible spherical tensorial algebra, i.e., by first re-expressing the Hamiltonians of section II C] as (following Ref. [63])

$$
\begin{aligned}
& \mathcal{H}_{n s r_{\mathrm{H}}}=c_{\mathrm{H}} \boldsymbol{T}^{(1)}\left(i_{\mathrm{H}}\right) \cdot \boldsymbol{T}^{(1)}(N), \\
& \mathcal{H}_{n s r_{\mathrm{D}}}=c_{\mathrm{D}} \boldsymbol{T}^{(1)}\left(i_{\mathrm{D}}\right) \cdot \boldsymbol{T}^{(1)}(N),
\end{aligned}
$$




$$
\begin{gathered}
\mathcal{H}_{d i p_{0}}=c_{\mathrm{S}} \boldsymbol{T}^{(1)}\left(i_{\mathrm{H}}\right) \cdot \boldsymbol{T}^{(1)}\left(i_{\mathrm{D}}\right), \\
\mathcal{H}_{\text {dip }}=g_{\mathrm{H}} g_{\mathrm{D}} \frac{\mu_{0} \mu_{N}^{2}}{4 \pi} \sqrt{10} \boldsymbol{T}^{(1)}\left(i_{\mathrm{H}}\right) \cdot \boldsymbol{T}^{(1)}\left(C^{2}, i_{\mathrm{D}}\right), \\
\mathcal{H}_{\text {quad }}=-e \boldsymbol{T}^{(2)}(Q) \cdot \boldsymbol{T}^{(2)}(\nabla E),
\end{gathered}
$$

with

$$
\boldsymbol{T}^{(1)}\left(i_{\mathrm{D}}, C^{2}\right)=\left[\boldsymbol{T}^{(1)}\left(i_{\mathrm{D}}\right) \otimes \boldsymbol{T}^{(2)}(C)\right]^{(1)},
$$

where the tensor $\boldsymbol{T}^{(2)}(C)$ is set for the spherical harmonic functions of second-order $Y_{2 q}$ associated with the rotation of the vibronic wave functions $\psi_{e v}$, from the laboratory-fixed to the molecule-fixed frame (see Appendix A) [64].

By employing the usual reduction rules on the tensorial products (cf. Appendix C of Messiah [65]), we can easily calculate the matrix elements (numerical applications are available in Appendix A).

\section{Nuclear Spin-Rotation Interactions}

In the chosen coupled basis (see section[IIB) the deuteron spin-rotation interaction $\mathcal{H}_{n s r_{\mathrm{D}}}$ [Eq. (4)] is purely diagonal, providing matrix elements independent of $F$ :

$$
\begin{aligned}
& \left\langle\psi_{e v} \Lambda^{\prime} ; N^{\prime} i_{\mathrm{D}}^{\prime} F_{1}^{\prime} i_{\mathrm{H}}^{\prime} F^{\prime} m_{F^{\prime}}\left|\mathcal{H}_{n s r_{\mathrm{D}}}\right| \psi_{e v} \Lambda ; N i_{\mathrm{D}} F_{1} i_{\mathrm{H}} F m_{F}\right\rangle \\
& =\left[F_{1}\left(F_{1}+1\right)-i_{\mathrm{D}}\left(i_{\mathrm{D}}+1\right)-N(N+1)\right] \frac{c_{\mathrm{D}}}{2} \delta_{F F^{\prime}} \delta_{F_{1} F_{1}^{\prime}} \delta_{N N^{\prime}} \delta_{i_{\mathrm{H}} i_{\mathrm{H}}} \delta_{i_{\mathrm{D}} i_{\mathrm{D}}^{\prime}} \delta_{m_{F} m_{F^{\prime}}} .
\end{aligned}
$$

On the other hand, the proton spin-rotation interaction $\mathcal{H}_{n s r_{\mathrm{H}}}$ [Eq. (3)] is not diagonal. After a double reduction, and by introducing the usual Wigner $6 \mathrm{j}$ symbols, we obtain the following matrix elements:

$$
\begin{aligned}
& \left\langle\psi_{e v} \Lambda^{\prime} ; N^{\prime} i_{\mathrm{D}}^{\prime} F_{1}^{\prime} i_{\mathrm{H}}^{\prime} F^{\prime} m_{F^{\prime}}\left|\mathcal{H}_{n s r_{\mathrm{H}}}\right| \psi_{e v} \Lambda ; N i_{\mathrm{D}} F_{1} i_{\mathrm{H}} F m_{F}\right\rangle \\
= & (-1)^{F+i_{\mathrm{H}}+i_{\mathrm{D}}+N^{\prime}+2 F_{1}+1} \sqrt{\left(2 F_{1}+1\right)\left(2 F_{1}^{\prime}+1\right)}\left\{\begin{array}{ccc}
F & i_{\mathrm{H}} & F_{1}^{\prime} \\
1 & F_{1} & i_{\mathrm{H}}
\end{array}\right\}\left\{\begin{array}{ccc}
i_{\mathrm{D}} & N^{\prime} & F_{1}^{\prime} \\
1 & F_{1} & N
\end{array}\right\} \\
& \times\left\langle N^{\prime}\left\|\boldsymbol{T}^{(1)}(N)\right\| N\right\rangle\left\langle i_{\mathrm{H}}^{\prime}\left\|\boldsymbol{T}^{(1)}\left(i_{\mathrm{H}}\right)\right\| i_{\mathrm{H}}\right\rangle c_{\mathrm{H}} \delta_{F F^{\prime}} \delta_{m_{F} m_{F^{\prime}}},
\end{aligned}
$$

which provides the selection rules: $\Delta N=\Delta F=\Delta m_{F}=0, \Delta F_{1}=0, \pm 1$ and $\Delta i_{\mathrm{H}}=\Delta i_{\mathrm{D}}=0$ (independently of $\Lambda$ ). 


\section{Spin-Spin Dipole Interactions}

The matrix elements of the scalar spin-spin interaction are readily calculated by a new double reduction. It becomes from Eq. [5]

$$
\begin{gathered}
\left\langle\psi_{e v} \Lambda^{\prime} ; N^{\prime} i_{\mathrm{D}}^{\prime} F_{1}^{\prime} i_{\mathrm{H}}^{\prime} F^{\prime} m_{F^{\prime}}\left|\mathcal{H}_{d i p_{0}}\right| \psi_{e v} \Lambda ; N i_{\mathrm{D}} F_{1} i_{\mathrm{H}} F m_{F}\right\rangle \\
=c_{s} \delta_{F^{\prime} F} \delta_{i_{\mathrm{H}}^{\prime} i_{\mathrm{H}}} \delta_{i_{\mathrm{D}}^{\prime} i_{\mathrm{D}}} \delta_{m_{F^{\prime}} m_{F}}(-1)^{F+F_{1}+i_{\mathrm{H}}}\left\{\begin{array}{ccc}
F & i_{\mathrm{H}} & F_{1}^{\prime} \\
1 & F_{1} & i_{\mathrm{H}}
\end{array}\right\}\left\langle i_{\mathrm{H}}^{\prime}\left\|\boldsymbol{T}^{(1)}\left(i_{\mathrm{H}}\right)\right\| i_{\mathrm{H}}\right\rangle \times \\
(-1)^{F_{1}^{\prime}+N^{\prime}+i_{\mathrm{D}}+1} \sqrt{\left(2 F_{1}+1\right)\left(2 F_{1}^{\prime}+1\right)}\left\{\begin{array}{ccc}
N^{\prime} & i_{\mathrm{D}} & F_{1}^{\prime} \\
1 & F_{1} & i_{\mathrm{D}}
\end{array}\right\}\left\langle i_{\mathrm{D}}^{\prime}\left\|\boldsymbol{T}^{(1)}\left(i_{\mathrm{D}}\right)\right\| i_{\mathrm{D}}\right\rangle \delta_{N^{\prime} N},
\end{gathered}
$$

which provides the same selection rules as the proton spin-rotation coupling [see the rules associated with Eq. [15].

The matrix elements of the second-order spin-spin dipole interaction [see Eq. [6] ] are obtained by a first reduction of the two tensors of first order, and then by a reduction of the term $\boldsymbol{T}^{(1)}\left(i_{\mathrm{D}}, C^{2}\right)$ to a product of a second order term $\boldsymbol{T}^{(2)}(C)$ by of a first order term $\boldsymbol{T}^{(1)}\left(i_{\mathrm{D}}\right)$. From Eq. [11), and by applying the property stemmed by Eq. [13, we calculate

$$
\begin{gathered}
\left\langle\psi_{e v} \Lambda^{\prime} ; N^{\prime} i_{\mathrm{D}}^{\prime} F_{1}^{\prime} i_{\mathrm{H}}^{\prime} F^{\prime} m_{F^{\prime}}\left|\boldsymbol{T}^{(1)}\left(C^{2}, i_{\mathrm{D}}\right) \cdot \boldsymbol{T}^{(1)}\left(i_{\mathrm{H}}\right)\right| \psi_{e v} \Lambda ; N i_{\mathrm{D}} F_{1} i_{\mathrm{H}} F m_{F}\right\rangle \\
=\delta_{F^{\prime} F} \delta_{m_{F^{\prime}} m_{F}}(-1)^{F+i_{\mathrm{H}}+F_{1}}\left\{\begin{array}{ccc}
F & i_{\mathrm{H}}^{\prime} & F_{1}^{\prime} \\
1 & F_{1} & i_{\mathrm{H}}
\end{array}\right\}\left\langle i_{\mathrm{H}}^{\prime}\left\|\boldsymbol{T}^{(1)}\left(i_{\mathrm{H}}\right)\right\| i_{\mathrm{H}}\right\rangle \\
\times\left\langle\psi_{e v} \Lambda^{\prime} ; N^{\prime} i_{\mathrm{D}}^{\prime} F_{1}^{\prime}\left\|\boldsymbol{T}^{(1)}\left(i_{\mathrm{D}}, C^{2}\right)\right\| \psi_{e v} \Lambda ; N i_{\mathrm{D}} F_{1}\right\rangle,
\end{gathered}
$$

with

$$
\begin{gathered}
\left\langle\psi_{e v} \Lambda^{\prime} ; N^{\prime} i_{\mathrm{D}}^{\prime} F_{1}^{\prime}\left\|\boldsymbol{T}^{(1)}\left(i_{\mathrm{D}}, C^{2}\right)\right\| \psi_{e v} \Lambda ; N i_{\mathrm{D}} F_{1}\right\rangle \\
=\left\langle\psi_{e v} \Lambda^{\prime} ; N^{\prime} i_{\mathrm{D}}^{\prime} F_{1}^{\prime}\left\|\left[\boldsymbol{T}^{(2)}(C) \otimes \boldsymbol{T}^{(1)}\left(i_{\mathrm{D}}\right)\right]^{(1)}\right\| \psi_{e v} \Lambda ; N i_{\mathrm{D}} F_{1}\right\rangle \\
=\sqrt{3\left(2 F_{1}+1\right)\left(2 F_{1}^{\prime}+1\right)}\left\{\begin{array}{ccc}
i_{\mathrm{D}} & i_{\mathrm{D}}^{\prime} & 1 \\
N & N^{\prime} & 2 \\
F_{1} & F_{1}^{\prime} & 1
\end{array}\right\} \\
\times\left\langle i_{\mathrm{D}}^{\prime}\left\|\boldsymbol{T}^{(1)}\left(i_{\mathrm{D}}\right)\right\| i_{\mathrm{D}}\right\rangle\left\langle\psi_{e v} \Lambda^{\prime} N^{\prime}\left\|\boldsymbol{T}^{(2)}(C)\right\| \psi_{e v} \Lambda N\right\rangle,
\end{gathered}
$$

where a Wigner 9j symbol has been introduced. By expressing the reduced vibronic matrix element in the molecule-fixed frame (using the second-rank rotation matrix)

$$
\begin{aligned}
& \left\langle\psi_{e \nu} \Lambda^{\prime} N^{\prime}\left\|\boldsymbol{T}^{(2)}(C)\right\| \psi_{e \nu} \Lambda N\right\rangle \\
= & \sqrt{(2 N+1)\left(2 N^{\prime}+1\right)} \sum_{q=-2}^{2}(-1)^{N^{\prime}-\Lambda^{\prime}}\left(\begin{array}{ccc}
N^{\prime} & 2 & N \\
-\Lambda^{\prime} & q & \Lambda
\end{array}\right)\left\langle\psi_{e \nu} \Lambda^{\prime}\left|\boldsymbol{T}_{q}^{(2)}(C)\right| \psi_{e \nu} \Lambda\right\rangle,
\end{aligned}
$$

where a Wigner 3j symbol appears, and where the molecular matrix element is defined by (with the help of the spherical 
harmonics)

$$
\left\langle\psi_{e v} \Lambda^{\prime}\left|\boldsymbol{T}_{q}^{(2)}(C)\right| \psi_{e v} \Lambda\right\rangle=\left\langle\psi_{e v} \Lambda^{\prime}\left|\frac{C_{q}^{(2)}(\theta, \phi)}{r_{\mathrm{HD}}^{3}}\right| \psi_{e v} \Lambda\right\rangle=2 \sqrt{\frac{\pi}{5}}\left\langle\psi_{e v} \Lambda^{\prime}\left|\frac{Y_{2 q}(\theta, \phi)}{r_{\mathrm{HD}}^{3}}\right| \psi_{e v} \Lambda\right\rangle .
$$

For coupling confined to a ${ }^{1} \Sigma$ electronic state $\left(\Lambda^{\prime}=\Lambda=q=0\right)$, the associated selection rules are: $\Delta F=0, \Delta F_{1}=0, \pm 1$, $\Delta N=0, \pm 2$, and $\Delta i_{\mathrm{H}}=\Delta_{\mathrm{D}}=0$. Thus the second-order spin-spin dipole coupling constant can be defined by

$$
c_{d i p}=g_{\mathrm{H}} g_{\mathrm{D}} \mu_{N}^{2} \frac{\mu_{0}}{4 \pi}\left\langle\psi_{e v}{ }^{1} \Sigma\left|\frac{1}{r_{\mathrm{HD}}^{3}}\right| \psi_{e v}{ }^{1} \Sigma\right\rangle
$$

obtained by factorizing the numerical coefficient of Eq. [11.

$\left\langle\frac{1}{r^{3}}\right\rangle$ is a traceless matrix for which only one component is required for a diatomic molecule.

\section{Quadrupolar Interaction}

The quadrupolar matrix elements are obtained by a usual reduction on Eq. (12). It becomes

$$
\begin{gathered}
\left\langle\psi_{e v} \Lambda^{\prime} ; N^{\prime} i_{\mathrm{D}}^{\prime} F_{1}^{\prime} i_{\mathrm{H}} F^{\prime} m_{F^{\prime}}\left|\boldsymbol{T}^{(2)}(\nabla E) \cdot \boldsymbol{T}^{(2)}\left(Q_{\mathrm{D}}\right)\right| \psi_{e v} \Lambda ; N i_{\mathrm{D}} F_{1} i_{\mathrm{H}} F m_{F}\right\rangle \\
=\delta_{F^{\prime} F} \delta_{m_{F^{\prime}} m_{F}} \delta_{F_{1}^{\prime} F_{1}}(-1)^{F_{1}^{\prime}+N+i_{\mathrm{D}}^{\prime}}\left\{\begin{array}{ccc}
F_{1} & N^{\prime} & i_{\mathrm{D}} \\
2 & i_{\mathrm{D}}^{\prime} & N
\end{array}\right\} \\
\times\left\langle\psi_{e v} \Lambda^{\prime} N^{\prime}\left\|\boldsymbol{T}^{(2)}(\nabla E)\right\| \psi_{e v} \Lambda N\right\rangle\left\langle i_{\mathrm{D}}^{\prime}\left\|\boldsymbol{T}^{(2)}\left(Q_{\mathrm{D}}\right)\right\| i_{\mathrm{D}}\right\rangle
\end{gathered}
$$

where the laboratory-fixed frame matrix element must be re-expressed in the molecule-fixed frame, i.e.,

$$
\begin{aligned}
& \left\langle\psi_{e v} \Lambda^{\prime} N^{\prime}\left\|\boldsymbol{T}^{(2)}(\nabla E)\right\| \psi_{e v} \Lambda N\right\rangle= \\
& \sqrt{\left(2 N^{\prime}+1\right)(2 N+1)} \sum_{q}(-1)^{N^{\prime}-\Lambda^{\prime}}\left(\begin{array}{ccc}
N^{\prime} & 2 & N \\
-\Lambda^{\prime} & q & \Lambda
\end{array}\right)\left\langle\psi_{e v} \Lambda^{\prime}\left|\boldsymbol{T}_{q}^{(2)}\left(\nabla E^{\prime}\right)\right| \psi_{e v} \Lambda\right\rangle .
\end{aligned}
$$

Equations (22) and (23) provide the selection rules: $\Delta F=\Delta F_{1}=0, \Delta N=0, \pm 1, \pm 2$, and $\Delta \Lambda=0, \pm 1, \pm 2$. When considering only the ground state of HD, the possible mixing between states with different values of $\Lambda$ can be ignored, because any electronic states with $\Lambda \neq 0$ (like the ${ }^{1} \Pi$ states) are located at energies very far away from the $X^{1} \Sigma$ state 66 .

Given $q=\Delta \Lambda=0$, the operator $\boldsymbol{T}_{0}^{(2)}\left(\nabla E^{\prime}\right)$ can be expressed as a function of $C_{0}^{(2)}(\theta, \phi)$ and of the spherical harmonics applied to the $n$ electron-nucleus coordinates, and then expressed in the Cartesian molecular coordinates as

$$
\left\langle\psi_{e v} \Lambda\left|\boldsymbol{T}_{0}^{(2)}\left(\nabla E^{\prime}\right)\right| \psi_{e v} \Lambda\right\rangle=-\frac{1}{2} \sum_{i}^{n} \frac{e_{i}}{4 \pi \epsilon_{0}}\left\langle\psi_{e v} \Lambda\left|\frac{3 z^{\prime^{2}}-r_{i}^{\prime^{2}}}{r_{i}^{\prime^{5}}}\right| \psi_{e v} \Lambda\right\rangle=-\frac{1}{2} \sum_{i}^{n} \frac{e_{i}}{4 \pi \epsilon_{0}}\left\langle\psi_{e v} \Lambda\left|\frac{\partial^{2}}{\partial z^{\prime^{2}}}\left(\frac{1}{r_{i}^{\prime}}\right)\right| \psi_{e v} \Lambda\right\rangle .
$$

Only the axial component of the electric field gradient tensor $\boldsymbol{V}$ (i.e., along the $z^{\prime}$ direction joining the two nuclei) has to be considered $\left(V_{x^{\prime} x^{\prime}}=V_{y^{\prime} y^{\prime}}=-V_{z^{\prime} z^{\prime}} / 2, \boldsymbol{V}\right.$ is a traceless tensor), and we define

$$
q_{\nu}=-\frac{1}{2}\left\langle\psi_{e v}^{1} \Sigma\left|V_{z^{\prime} z^{\prime}}\right| \psi_{e v}^{1} \Sigma\right\rangle
$$


Table I. Vibrational dependence of the hyperfine constants of the ground electronic state of HD 49].

\begin{tabular}{|c|c|c|c|c|c|c|}
\hline $\begin{array}{c}\text { Vibrational } \\
\text { Quantum } \\
\text { Number }\end{array}$ & $\begin{array}{c}\text { Energies } \\
\left(\mathrm{cm}^{-1}\right)\end{array}$ & $\begin{array}{c}c_{\mathrm{H}} \\
(\mathrm{kHz})\end{array}$ & $\begin{array}{c}c_{\mathrm{D}} \\
(\mathrm{kHz})\end{array}$ & $\begin{array}{c}c_{\text {dip }} \\
(\mathrm{kHz})\end{array}$ & $\begin{array}{c}c_{\text {quad }}=e q_{\nu} Q_{\mathrm{D}} \\
(\mathrm{kHz})\end{array}$ & $\begin{array}{c}c_{s} \\
(\mathrm{~Hz})\end{array}$ \\
\hline \hline \multirow{2}{*}{0} & 0 & $85.600(18)[47$ & $13.122(11)[47]$ & $44.403(30)[47$ & $224.54(6)[47]$ & $47(7)[67]$ \\
\cline { 3 - 7 } & 86.2832 & 13.2450 & 44.5792 & 226.6493 & $43(41.1[15])$ \\
\hline \multirow{2}{*}{1} & 3633.0 & 85.0775 & 13.0599 & 43.2106 & 225.0968 & \\
\cline { 2 - 7 } & & $(84.404)$ & $(12.939)$ & $(43.040)$ & $(223.00)$ & \\
\cline { 2 - 7 } & 7088.9 & 83.5670 & 12.8280 & 41.7974 & 222.5516 & \\
\cline { 2 - 7 } & & $(82.905)$ & $(12.709)$ & $(41.632)$ & $(220.480)$ & \\
\hline
\end{tabular}

The values in parentheses $(v=2)$ are corrected from the difference with the values obtained from the experimental data $(N=1, v=0)$, see text.

${ }^{\dagger}$ Anharmonic energies (harmonic energy: $3815.10 \mathrm{~cm}^{-1}$ ).

which is a scalar quantity independent of $N$ and $N^{\prime}$. This quantity can be related to the electric field gradient at the deuteron $q_{\mathrm{D}} 67,68$.

The second reduced matrix element of Eq. 22) is given in Appendix A for the usual definition of the deuteron quadrupole moment $Q_{\mathrm{D}}$, This let us derive the common hyperfine quadrupolar constant $c_{q u a d}=e q_{\nu} Q_{\mathrm{D}}$.

\section{E. Eigenenergies, Wave functions and Assignment}

The matrix elements provided by section II D are implemented in a $\left(2 i_{\mathrm{D}}+1\right)\left(2 i_{\mathrm{H}}+1\right) \times\left(2 i_{\mathrm{D}}+1\right)\left(2 i_{\mathrm{H}}+1\right)$ square matrix after calculating the respective Wigner symbols, with the help of the selection rules associated with the coupled basis vector established previously (see Fig. 1). Actually, each matrix element is a diagonal blocked sub-matrix of size $(2 F+1) \times(2 F+1)$. The most comprehensive matrix elements (i.e., $\Lambda \neq 0)$ have been numerically implemented.

As previously pointed out, the hyperfine matrix elements $\Delta N= \pm 2$ (assigned by an $\mathbf{X}$ without parentheses in Fig. 1 ) barely contribute to the coupling when their value is small compared with the energy difference between the two coupled rotational levels. This is particularly suitable when the rotational constants are large, like for HD. In that case, the $\Delta N= \pm 2$ couplings can be ignored, and only the squared-frame matrix elements of Fig. 1 matter. In this specific case, a unitary transformation (here, a double permutation of the two central columns and rows) allows for diagonalizing two trivial $2 \times 2$ matrices along $\Delta N=0$ instead of diagonalizing one $\left(2 i_{\mathrm{D}}+1\right)\left(2 i_{\mathrm{H}}+1\right) \times\left(2 i_{\mathrm{D}}+1\right)\left(2 i_{\mathrm{H}}+1\right)$ matrix (see Fig.1]. The net result is that $N$ remains a quasi good quantum number. However, since each value of $N$ can be coupled up to two values of $F_{1}$, we have introduced the label \pm when necessary, i.e., “+” when $N=F_{1}$, and “-” otherwise (following spin-rotation conventional notation).

\section{F. Comparison with previous works}

Four values of the hyperfine constants of the ground vibrational state $(v=0)$ have been proposed by Ramsey and coauthors in two papers 46 47. They have been obtained from energy level splitting of the Zeeman sub-transitions under magnetic field inside the $N=1$ rotational level, see Table T The scalar spin-spin dipole constant $c_{s}$ is barely considered here, because it is approximately three orders of magnitude weaker than the other constants [15, 67, 69, 70]. It is worth noting that Ramsey and co-authors 46, 47 defined the two hyperfine constants $d_{1}=2 c_{d i p} / 5$ and $d_{2}=\frac{-e q_{v} Q_{\mathrm{D}}}{10}$. 


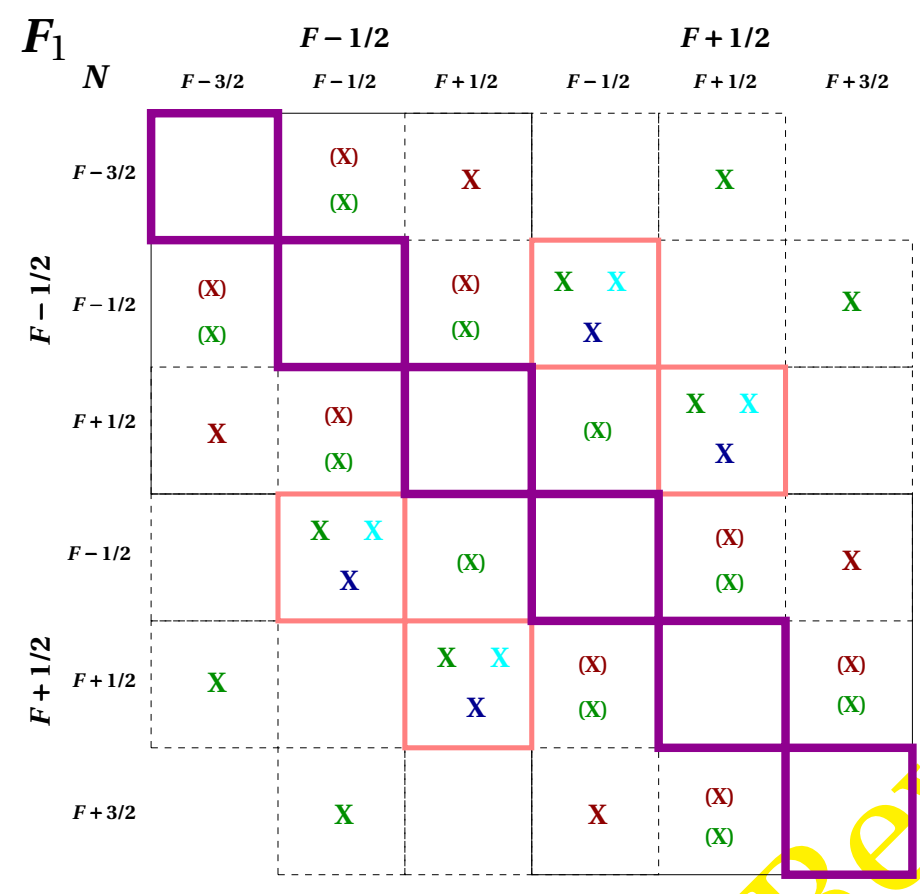

$$
\begin{aligned}
& \mathscr{H}_{n s r_{\mathrm{D}}}: \Delta N=0 ; \Delta F_{1}=0 \\
& \mathscr{H}_{n s r_{\mathrm{H}}}: \Delta N=0 ; \Delta F_{1}=0, \pm 1 \\
& \mathscr{H}_{\text {quad }}: \Delta N=0,( \pm 1) \pm 2 ; \Delta F_{1}=0 \\
& \mathscr{H}_{\text {dip }}: \Delta N=0,( \pm 1), \pm 2 ; \Delta F_{1}=0, \pm 1 \\
& \mathcal{H}_{\text {di } p_{0}}: \Delta N=0 ; \Delta \hat{F}_{1}=0, \pm 1
\end{aligned}
$$

(a)

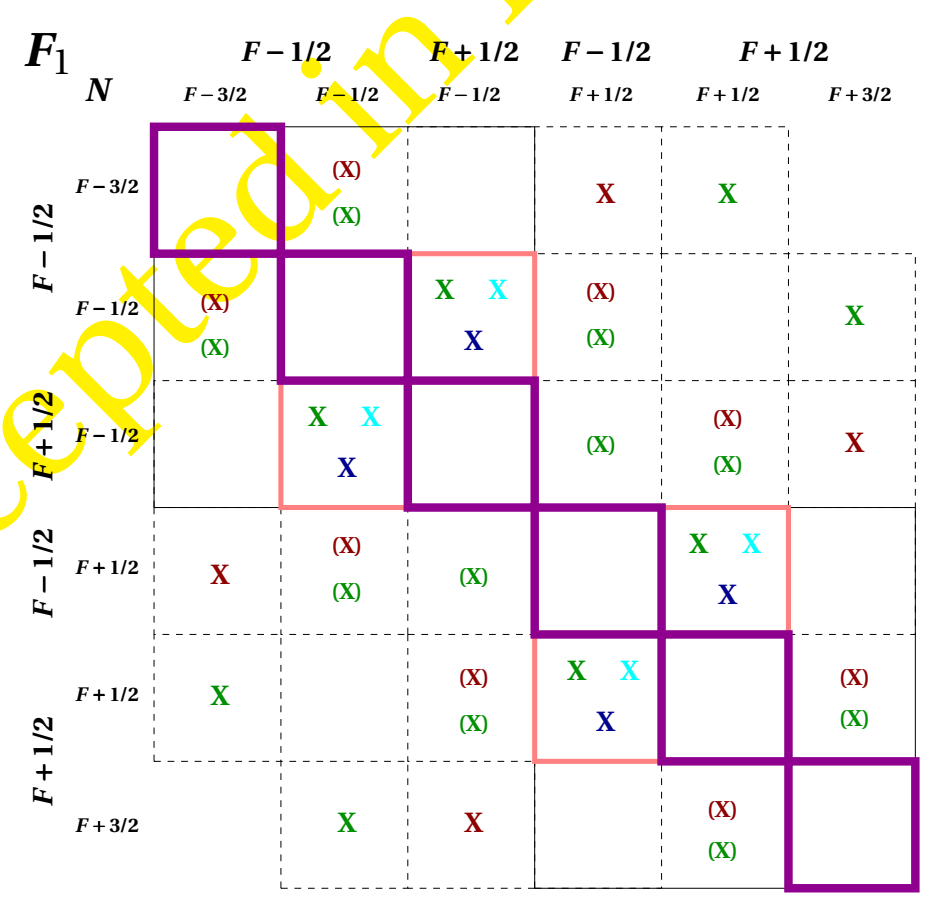

(b)

Figure 1. Hyperfine matrices (HD-like, i.e., $\Delta i_{\mathrm{D}}=\Delta i_{\mathrm{H}}=\Delta F=0, i_{\mathrm{H}}=1 / 2, i_{\mathrm{D}}=1$ ). Sub-figure a): crude hyperfine matrix for linear molecules. The $(\mathbf{X})$ notation indicates the off-diagonal matrix elements $\Delta N= \pm 1$ which are null for $\Lambda=0\left({ }^{1} \Sigma\right.$ state). The framed matrix elements follow $\Delta N=0$. The selection rules are indicated for each coupling, and coordinated by the color set. The diagonal matrix elements $\left(\Delta N=\Delta F_{1}=0\right)$ comprise the rotational energy (purely diagonal) and the diagonal hyperfine matrix elements. Sub-figure b): matrix obtained after a unitary transformation (permutation of two lines and columns), see text. 


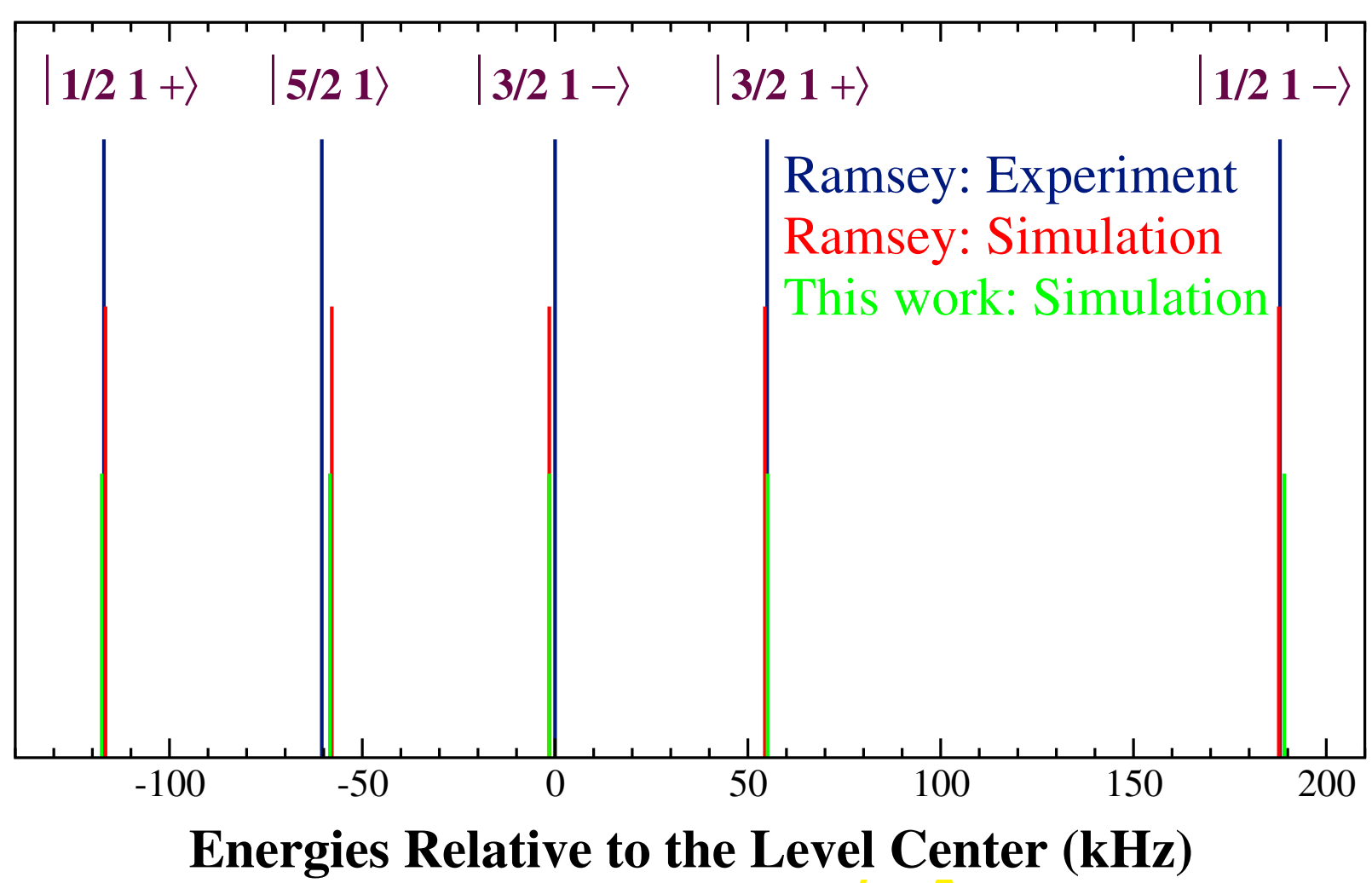

Figure 2. Hyperfine structure of HD for the level $N=1, v=0$. The level assignment $|F N=1 \pm\rangle$ is defined in section IIE (it is noteworthy that the assignment \pm differs from that employed by Ramsey). The experimental energies are displayed in dark blue, from Ref. [46. The calculated energies obtained from the Ramsey constants [46] are displayed in red, and those obtained from the $a b$ initio constants are displayed in green (see Table I. The overlap is better than $3 \mathrm{kHz}$. The rotational constants have been extracted from Ref. [1].

We observe that the calculated constants in this work are slightly different (i.e., within $1 \%$, that is approximately one order of magnitude larger than the experimental accuracy) from those extrapolated from the experiment, when they can be compared (i.e., for $v=0$, no other values are available in the literature). Nevertheless, the most important result is the behavior of the constants versus the vibrational mode considered: decreasing values with the vibrational energy growth. Hence the values used for the energy calculation of the vibrational level $v=2$ have been corrected for these differences (i.e., extrapolated from $v=0$ ); see Table $]$

\section{G. Validation}

The correctness of the matrix element calculation has been checked by reproducing the experimental data available in the literature, i.e., the hyperfine level energies obtained under magnetic field (it is worth noting that the hyperfine constants provided by Ramsey and co-authors 46 47 have been evaluated by using a decoupled basis-set). The energy values have been extrapolated to zero magnetic field from Fig. 1 of Ref. [46] with the best accuracy possible. They are reported in Fig. 2 and compared to our predictions based on the Ramsey's hyperfine coupling values, as well as on our ab initio values (for $v=0$ ).

It is worth noting that we reach an almost perfect agreement between the calculated and the experimental energies, knowing that the experimental energies extracted from Ref. 46] suffer from evaluated uncertainties of the order of $\pm 2.5 \mathrm{kHz}$, which is inside the uncertainty range of our determinations (after correction of the constants). We anticipate 
Table II. Herman-Wallis coefficients of HD for the vibrational band $v: 2 \leftarrow 0$.

\begin{tabular}{|c|c|c|}
\hline $\begin{array}{c}\mu_{0} \\
(\mu \mathrm{D})\end{array}$ & $c_{1}$ & $c_{2}$ \\
\hline \hline $19.35(12)$ & $0.0569(30)$ & $-2.00(39) \times 10^{-3}$ \\
\hline
\end{tabular}

The Herman-Wallis coefficients [74] have been obtained by fitting the data provided by Kassi and Campargue[73] with a uniform data weighting: $\mu(m)=\mu_{0}\left(1+c_{1} m+c_{2} m^{2}\right)$, with $m=N+1$ for the $R$ branch, and $m=-N$ for the $P$ branch (this factor can be deduced from formula B7 by setting $\Lambda=0$ ).

The uncertainties (three times the standard deviation) are provided in parentheses (unit of the last digits).

that a similar uncertainty must affect the relative energy positions of the two other vibrational levels. However, there is currently no experimental data to validate this estimation.

\section{LINE INTENSITIES}

\section{A. Implementation}

The calculation of the matrix elements, including the rotational energies and the hyperfine couplings, the diagonalization of the full matrix (i.e., without unitary transformation or approximation, see section IIE), the recovering of the level assignments, and then, the calculation of the linear line intensity have been implemented in the application "STEPRAM". This application is based on the language perl calling "c" subroutines. The matrix diagonalization algorithm is provided by the package PDL (based on LAPACK) [72].

\section{B. Electric Dipole Moment}

Strictly considering, the weak rovibrational transitions inside the ground state of HD follow the selection rules and labeling associated with the symmetry group $C_{\infty h}[29$. However, because of the weak $u / g$ mixing in a nonadiabatic BOA, the usual symmetry notation of the BOA wave function can be conserved.

The calculation of the line intensity of the hyperfine transitions can be deduced from known vibration-rotation line intensities. Actually, Ref. [73] provides a list of experimental line intensities, and of electric dipole moments for 11 rotational lines of the first overtone band at 294 (1) K. Indeed, the observed values are very close to the calculated ones (within 1\%) [25]. For the sake of completeness, the experimental intensities have been fitted (unweighted data) to obtain the Herman-Wallis coefficients [74] of the first overtone band (see Table II]. It is worth pointing out that the variation of the dipole moment of the overtone with the rotational transition results from the centrifugal distortion (rotation-vibration interaction) which is a diagonal correction to the $\mathrm{BOA}[29]$.

\section{Linear Dipolar Line Intensity}

Following the usual definition of the line intensity set for degenerate transitions (following HITRAN), we define [75] 


$$
S_{u l}=\sum_{m_{u}} \sum_{m_{l}} S_{u m_{u} ; l m_{l}}=\frac{2 \pi^{2}}{3 c h \epsilon_{0}} \tilde{\sigma}_{u l} \frac{\mathrm{e}^{-\frac{E_{l}}{k T}}-\mathrm{e}^{-\frac{E_{u}}{k T}}}{Q(T)}\left|\mu_{u l}\right|^{2}
$$

where $c, \epsilon_{0}, k$ and $h$ are the usual constants of the International System of Units, where $Q(T)$ is the partition function, $E_{u}$ and $E_{l}$ are the energies of the upper and lower levels, respectively, $\tilde{\sigma}_{u l}$ is the wavenumber of the hyperfine transition, and $\mu_{u l}$ is the dipole moment of the hyperfine transition in the electric dipole approximation (see Appendix B). The isotopic abundance is set to 1 (at the opposite of HITRAN). $S_{u m_{u} ; l m_{l}}$ are the line intensities of the sub-transition associated with each upper $\left|u m_{u}\right\rangle$ and lower $\left|l m_{l}\right\rangle$ Zeeman degenerate sub-levels. Actually, $\left|l m_{l}\right\rangle$ is set for the eigenvector $\left|\begin{array}{c}\Lambda_{l}, F_{l}, m_{F_{l}}, i_{\mathrm{H}_{l}}, i_{\mathrm{D}_{l}},\left(N_{l}, F_{1_{l}}\right) \\ \psi_{e \nu_{l}}\end{array}\right\rangle$, and $\left|u m_{u}\right\rangle$ for $\left|\psi_{e v_{u}}^{\Lambda_{u}, F_{u}, m_{F_{u}}, i_{\mathrm{H}_{u}}, i_{\mathrm{D}_{u}},\left(N_{u}, F_{1_{u}}\right)}\right\rangle$ which are obtained by matrix diagonalization (see section II E. By introducing the mixing coefficients $a_{N, F_{1}}^{\Lambda, F, i_{\mathrm{H}}, i_{\mathrm{D}},\left(N, F_{1}\right)}$ (derived from the diagonalization), the wave functions are defined by

$$
\left|\psi_{e v}^{\Lambda, F, m_{F}, i_{\mathrm{H}}, i_{\mathrm{D}},\left(N, F_{1}\right)}\right\rangle=\sum_{F_{1}=\left|F-i_{\mathrm{H}}\right|}^{F+i_{\mathrm{H}}} \sum_{N=\left|F_{1}-i_{\mathrm{D}}\right|}^{F_{1}+i_{\mathrm{D}}} a_{N, F_{1}}^{\Lambda, F, i_{\mathrm{H}}, i_{\mathrm{D}},\left(N, F_{1}\right)}\left|\psi_{e \nu} \Lambda ; N i_{\mathrm{D}} F_{1} i_{\mathrm{H}} F m_{F}\right\rangle
$$

where the assignment $\left(N, F_{1}\right)$ is set for $(N, \pm)$, as discussed in section II E

Here, we focus our attention on the hyperfine line intensity (i.e., $S_{u l}$ ), and we mainly ignore the Zeeman subline intensity (i.e., $S_{u m_{u} ; l m_{l}}$ ). However, knowing $S_{u l}$, it is easy to deduce these subline intensities $S_{u m_{u} ; l m_{l}}$ when the impinging beam polarization (associated with $\Delta m_{F}=0, \pm 1$ ) is known since

$$
S_{u m_{u} ; l m_{l}}=\left(\begin{array}{ccc}
F_{u} & 1 & F_{l} \\
-m_{F_{u}} & \Delta m_{F} & m_{F_{l}}
\end{array}\right)^{2} S_{u l} \text {. }
$$

Actually, the values of intensities $S_{u m_{u} ; l m_{l}}$ are required when dealing with nonlinear absorption.

\section{Stick Spectra}

Armed by the formalism described in the Appendix B, we can generate the line intensity of the hyperfine transitions under interests [i.e., by evaluating the quantity $S_{u l}$, see Eq. 26] ], and then plot the generated stick spectrum for various rotational transitions (the couplings $\Delta \Lambda \neq 0$ can be ignored, because the $B^{1} \Sigma_{u}^{+}$, and $C^{1} \Pi_{u}$ states are far away 76 ). The simulations have been currently performed for two rotational transitions: $P_{1}$ (Fig. 3 ) and $R_{1}$ (Fig. 4) of the first overtone of $\mathrm{HD}$, at room temperature (the two lines share the same lower levels). In addition to the values of the four relevant hyperfine constants, the value of the rotational and of the centrifugal distortion constants are required. The values of $B, D$ and $H$ constants have been taken from the literature: for the ground state in Ref. [71], and for the upper state (including the band origin) in Ref. [73]. The dipole moments of the calculated transitions follow the values provided by section III B. For each simulation, the center of gravity of the hyperfine pattern has been shifted to match the relevant rotational transition center. Hence the Doppler-broadened profile (under linear absorption) of the respective transition 


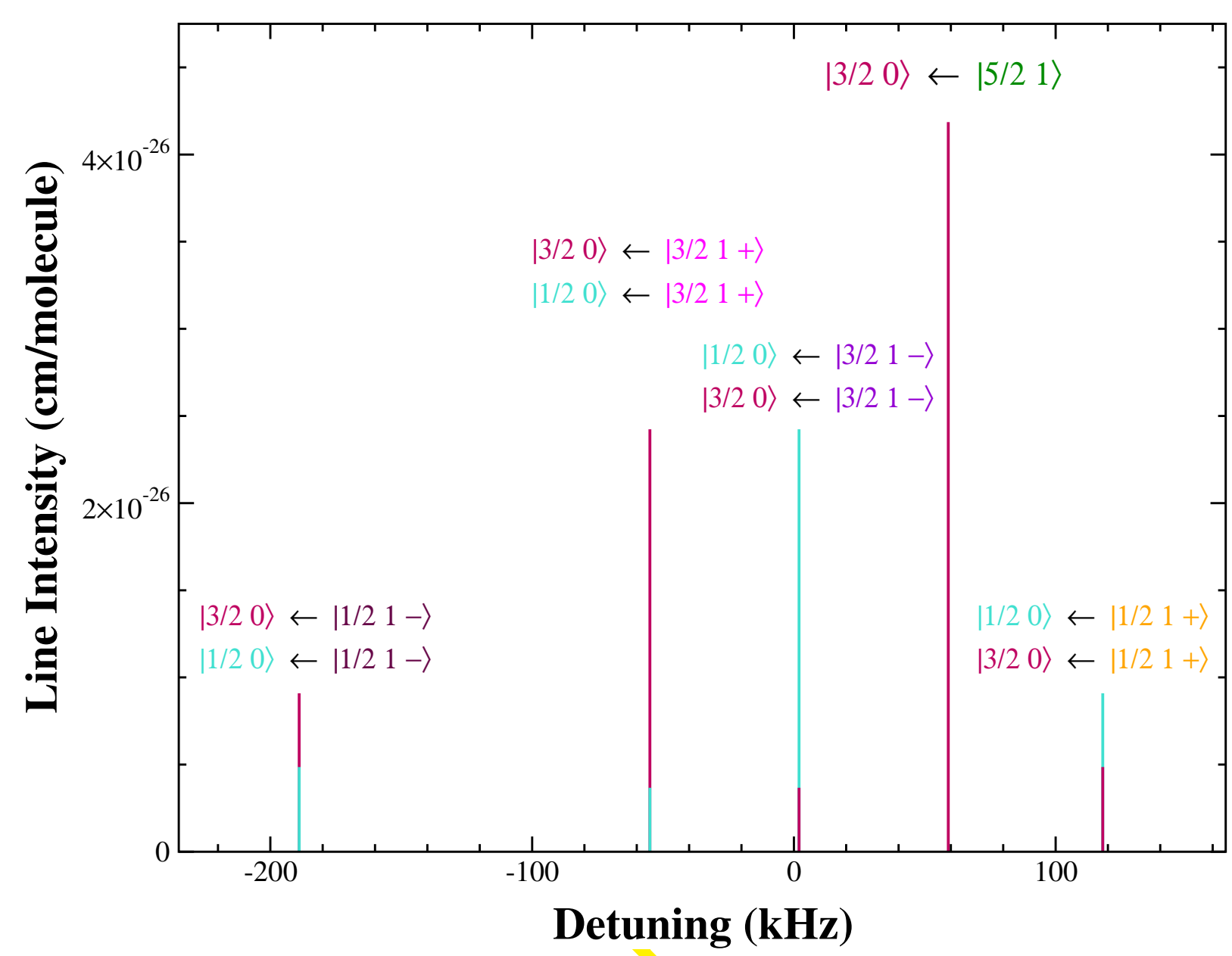

Figure 3. Line intensities of the transition $P_{1}$. The pattern is centered on the center of gravity of the rovibrational transition $(209,7842,90.882 \mathrm{MHz}$ ). The partition function is set to 29.8744 (i.e., for $296 \mathrm{~K}$ ). The dipole moment is set to $18.2 \mu \mathrm{D}$ (according to Table II. The rotational constants are taken from Ulivi, de Natale, and Inguscio 71 for the ground vibrational state, and from Kassi and Campargue 73 for the upper vibrational state. The energy level assignment is identical to that provided on Fig. 2 A color coding is associated with the energy levels and to the transitions.

can be centered at a null detuning.

Figure 3 exhibits a spectrum spreading over $\sim 300 \mathrm{kHz}$ (nine hyperfine lines) while Fig. 4 exhibits an extension approximately twice larger (21 hyperfine lines). Both spectra are dominated by the strongest (and nondegenerate) line issued from the same hyperfine level $\left|F_{l}=5 / 2, N_{l}=1\right\rangle$ (there is only one level $F=5 / 2$ for $N=1$ ). The other hyperfine lines of the transition $P_{1}$ are doublets, because the levels $F=1 / 2$ and $F=3 / 2$ are degenerate for $N=0$. The stick spectrum of the other rotational transitions $(N<6)$ has also been analyzed (not shown), the spectral extension slightly increases with $N$. These extensions can approach $1 \mathrm{MHz}$. It is worth noting that the strongest hyperfine lines tend to spread over $\sim 100 \mathrm{kHz}$ for each rotational transition.

The spectrum of the transition $R_{1}$ looks very different from that of the transition $P_{1}$, because of the larger number of hyperfine lines, and also because of the lift of hyperfine level degeneracies. Actually, the central frequency of the transition $R_{1}$ has been recently determined within a few tens of $\mathrm{kHz}$ accuracy, by two different groups using sub-Doppler techniques [16, 17], but they provide two incompatible determinations, while only the Amsterdam group reported about possible contributions of the hyperfine splittings on the observed unresolved resonance profile [16]. 


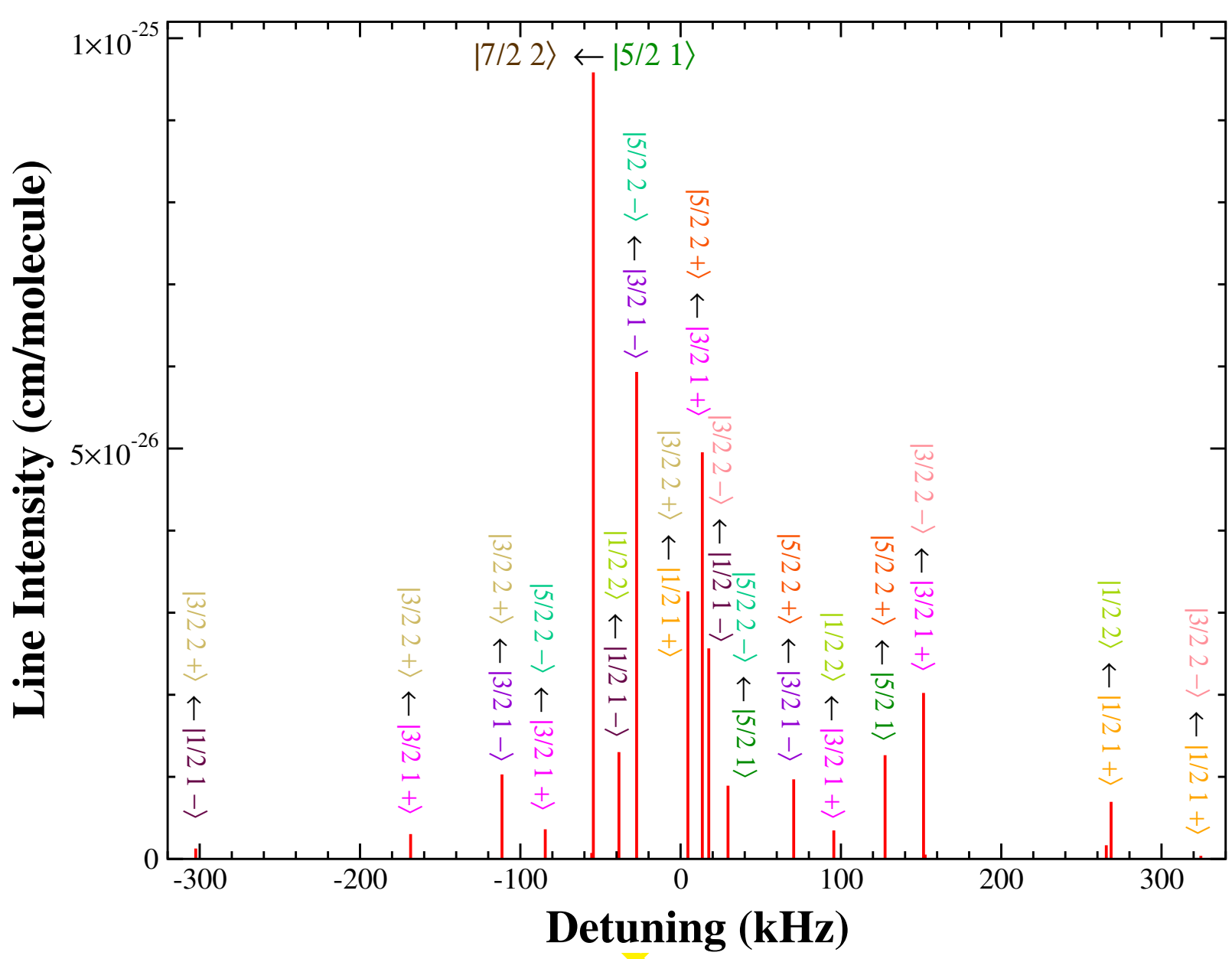

Figure 4. Line intensities of the transition $R_{1}$. The pattern is centered on the center of gravity of the rovibrational transition $(217,105,183.925 \mathrm{MHz}$ ). The partition function is set to 29.8744 (at $296 \mathrm{~K}$ ). The dipole moment is set to $21.4 \mu \mathrm{D}$ (according to Table II). Only the assignment of the lines with an intensity larger than $1.5 \times 10^{-27} \mathrm{~cm} / \mathrm{mol}$. is reported over a total of 21 hyperfine lines. A color coding is associated with the energy levels. The rest of the caption is identical to that of Fig. 3

\section{DISCUSSION}

Given our simulations of the hyperfine line patterns (Figs. 3 and 4 ), the pivotal inquires are, (i) is it possible to observe the predicted hyperfine components, and (ii) which "artifacts" would be induced under non-linear absorption experiments since, 1) only a sub-Doppler technique can allow for approaching the required spectral resolution, and 2) only a very sensitive technique can exhibit a sufficient signal-to-noise ratio? Moreover, nonlinear absorption and high sensitivity do not support a trivial congruent. Nowadays, both targeted objectives (resolution and sensitivity) are challenging for the most elaborated absorption techniques: CRDS (Cavity RingDown Spectroscopy) [17] 77], NICE-OHMS (Noise-Immune Cavity-Enhanced Optical Heterodyne Spectroscopy) [16] or any CEAS (Cavity Enhanced Absorption Spectroscopy). Additionally, the challenge is even more severe when we consider that nonlinear couplings (associated with saturated saturation) would induce crossover resonances, i.e., nonlinear resonances involving more than two energy levels, and even the (degenerated) Zeeman sublevels. Furthermore, additional sideband crossover resonances (78) can be anticipated when a frequency modulation technique is adopted.

While our calculations have been focused on the key first overtone of HD, they can be easily performed for any other vibrational transitions. Actually, the study of the fundamental overtone may be also very promising. The line 
intensities can readily be deduced by using the constants provided in Table $\mathbb{1}$ Here, we disregarded about any possible experimental issues which require for specific attention. Nevertheless, it seems that the use of a magnetic field could help to resolve the hyperfine components, at least partially, because it would spread the hyperfine transitions over a larger spectral pattern.

Of course, the quality of the predictions on the relative position of the line position needs to be experimentally investigated. The transition $R_{1}$ (Fig. 4) shows a few hyperfine transitions separated by less than $10 \mathrm{kHz}$, which is currently a real experimental challenge. However, the transitions $R_{0}$ and $P_{1}$ (Fig. 3 let us anticipate more favorable conditions despite the hyperfine line overlapping, because (i) the minimum frequency separation between transitions reaches $\sim 60 \mathrm{kHz}$, and (ii) the number of distinguishable hyperfine transitions is limited to five with three ones prevailing (the pattern of the transition $R_{0}$ is very similar to that of the transition $P_{1}$, except that the frequency scale is reversed). These reduced structures greatly simplify the calculation of the spectral pattern under saturated absorption since only a two-level $\Lambda$ or $V$ coupling scheme is involved.

It is worth pointing out that our approach of the calculation of the hyperfine line intensity is certainly not ultimate in the absence of calculations of all the hyperfine couplings at the best computational level which would include a nonadiabatic BOA or a Post-BO approach (see Introduction), and relativistic corrections. In this first tentative, the hyperfine parameters have been calculated by using a pure adiabatic BOA, but only minuscule corrections are anticipated (see section II A inaccuracies are provided). However, the calculated hyperfine level energies have been positively checked with Ramsey's data when it was possible. Thus we anticipate only little propagation of the modeling deficiencies to the hyperfine line intensities.

Last, although the hyperfine formalism has been specifically discussed for the ground electronic state of HD, it has been implemented to cover the upper electronic states of HD exhibiting non null values of $\Lambda$ and the cases of any linear molecule in the singlet state exhibiting two different nuclear spins; $\mathrm{cf}$. $\mathrm{C}_{2} \mathrm{HD}$, DF, CsF, and even $\mathrm{C}^{17} \mathrm{O}$ or ${ }^{16} \mathrm{OC}{ }^{17} \mathrm{O}$ (molecules exhibiting a single nuclear spin).

\section{CONCLUSIONS}

By considering the hyperfine structure of linear molecules involving two different values of the nuclear spin with one $>1 / 2$, and by quantum-chemical calculations of the HD hyperfine constants, we have first validated the predicted level structure via the data available in the literature. We then established a formalism allowing us to calculate the linear line intensity of the hyperfine transitions, key data for nonlinear sub-Doppler spectroscopy calculations. In that perspective, the Herman-Wallis coefficients have been derived from the data available in literature. The hyperfine stick spectra have been mainly discussed for two transitions belonging to the first overtone band of HD (i.e., the transitions $P_{1}$ and $R_{1}$ ). They show spectral extensions which can reach $1 \mathrm{MHz}$, opening opportunities to analyze data issued from extremely accurate and sensitive experimental setups.

\section{ACKNOWLEDGMENTS}

The author wishes to sincerely thank Jürgen Gauss for running the calculation of the hyperfine constants. 


\section{APPENDICES}

A. Some key matrix elements associated with $i_{\mathrm{H}}=1 / 2, i_{\mathrm{D}}=1$

$$
\begin{aligned}
& \left\langle i_{\mathrm{H}}^{\prime}\left\|\boldsymbol{T}^{(1)}\left(i_{\mathrm{H}}\right)\right\| i_{\mathrm{H}}\right\rangle=\delta_{i_{\mathrm{H}} i_{\mathrm{H}}^{\prime}} \sqrt{i_{\mathrm{H}}\left(i_{\mathrm{H}}+1\right)\left(2 i_{\mathrm{H}}+1\right)}=\sqrt{\frac{3}{2} .} \\
& \left\langle i_{\mathrm{D}}^{\prime}\left\|\boldsymbol{T}^{(1)}\left(i_{\mathrm{D}}\right)\right\| i_{\mathrm{D}}\right\rangle=\delta_{i_{\mathrm{D}} i_{\mathrm{D}}^{\prime}} \sqrt{i_{\mathrm{D}}\left(i_{\mathrm{D}}+1\right)\left(2 i_{\mathrm{D}}+1\right)}=\sqrt{6} .
\end{aligned}
$$

$$
\left\langle i_{\mathrm{D}}^{\prime}\left\|\boldsymbol{T}^{(2)}\left(Q_{\mathrm{D}}\right)\right\| i_{\mathrm{D}}\right\rangle=\frac{Q_{\mathrm{D}}}{2} \sqrt{\frac{\left(i_{\mathrm{D}}+1\right)\left(2 i_{\mathrm{D}}+1\right)\left(2 i_{\mathrm{D}}+3\right)}{i_{\mathrm{D}}\left(2 i_{\mathrm{D}}-1\right)}}=\sqrt{\frac{15}{2}} Q_{\mathrm{D}},
$$

with $Q_{\mathrm{D}}=2\left\langle i_{\mathrm{D}}, m_{i_{\mathrm{D}}}=i_{\mathrm{D}}\left|\boldsymbol{T}_{0}^{(2)}\left(Q_{\mathrm{D}}\right)\right| i_{\mathrm{D}}, m_{i_{\mathrm{D}}}=i_{\mathrm{D}}\right\rangle[79$.

\section{B. Calculation of $\left|\mu_{u l}\right|^{2}$}

Commonly dealing with the linear absorption approximation resulting from an electric dipole transition, it is relevant to survive the Hamiltonian coupling the external electromagnetic field $E(t)$ (in the slowly varying envelop approximation) with the hyperfine transition electric dipole moment $\mu_{u l}$ as defined in section III C. By using a similar tensorial formalism to that introduced in section II we define, in the laboratory-fixed frame

$$
\mathcal{H}_{e 1}(t)=-\boldsymbol{T}^{(1)}(\mu) \cdot \boldsymbol{T}^{(1)}(E)=E(t) \sum_{q}(-1)^{q} \boldsymbol{T}_{q}^{(1)}(\mu) \boldsymbol{T}_{-q}^{(1)}(\epsilon)
$$

where $\boldsymbol{T}^{(1)}(\epsilon)$ is a normalized to one (unitless), possibly complex quantity whose tensorial components $(q)$ reflect the direction of the polarization of the external EMF, $E(t)$ being a scalar. Thus only the matrix elements of $\boldsymbol{T}_{q}^{(1)}(\mu)$ need to be calculated. It becomes by reduction

$$
\begin{aligned}
\mu_{u l_{q}} & =\left\langle\psi_{e v_{u}}^{\Lambda_{u}, F_{u}, m_{F_{u}}, i_{\mathrm{H}_{u}}, F_{1_{u}}\left(i_{\mathrm{D}_{u}}, N_{u}\right)}\left|\boldsymbol{T}_{q}^{(1)}(\mu)\right| \psi_{e v_{l}}^{\Lambda_{l}, F_{l}, m_{F_{l}}, i_{\mathrm{H}_{\mathrm{l}}}, F_{\mathrm{l}_{l}}\left(i_{\mathrm{D}_{l}}, N_{l}\right)}\right\rangle \\
& =(-1)^{F_{u}-m_{F_{u}}}\left(\begin{array}{ccc}
F_{u} & 1 & F_{l} \\
-m_{F_{u}} & q & m_{F_{l}}
\end{array}\right)\left\langle\psi_{e v_{u}}^{\Lambda_{u}, F_{u}, i_{\mathrm{H}_{u}}, F_{1_{u}}\left(i_{\mathrm{D}_{u}}, N_{u}\right)}\left\|\boldsymbol{T}^{(1)}(\mu)\right\| \psi_{e v_{l}}^{\left.\Lambda_{l}, F_{l}, i_{\mathrm{H}_{\mathrm{l}}}, F_{1_{l}}\left(i_{\mathrm{D}_{l}}, N_{l}\right)\right\rangle}\right\rangle \\
& =(-1)^{F_{u}-m_{F_{u}}}\left(\begin{array}{ccc}
F_{u} & 1 & F_{l} \\
-m_{F_{u}} & q & m_{F_{l}}
\end{array}\right) \mu_{u l} .
\end{aligned}
$$


By inserting the mixing coefficients [see Eq. [27] into Eq. [B2], by multiplying by the complex conjugated expression, and by summing over all the polarization components $\left(\Delta m_{F}=0, \pm 1\right)$, we obtain [80] [following Eq. 26] ]

$$
\begin{aligned}
& \left|\mu_{u l}\right|^{2}=\mid \sum_{F_{1_{l}}=\left|F_{l}-i_{\mathrm{H}_{l}}\right|}^{F_{l}+i_{\mathrm{H}_{l}}} \sum_{F_{1_{u}}=\left|F_{u}-i_{\mathrm{H}_{u}}\right|}^{F_{u}+i_{\mathrm{H}_{u}}} \sum_{N_{l}=\left|F_{1_{l}}-i_{\mathrm{D}_{1}}\right|}^{F_{1_{l}}+i_{\mathrm{D}_{l}}} \sum_{N_{u}=\left|F_{1_{u}}-i_{\mathrm{D}_{u}}\right|}^{F_{1_{u}}+i_{\mathrm{D}_{u}}} \\
& \left.a_{N_{l}, F_{l}, i_{\mathrm{H}_{l}}, i_{\mathrm{D}_{l}},\left(N_{l}, F_{1_{l}}\right)} a_{N_{u}, F_{1_{u}}}^{\Lambda_{u}, F_{u}, i_{\mathrm{H}_{u}}, i_{\mathrm{D}_{u}},\left(N_{u}, F_{1_{u}}\right)}\left\langle\psi_{e v_{u}} \Lambda_{u} ; i_{\mathrm{D}_{u}} N_{u} F_{1_{u}} i_{\mathrm{H}_{u}} F_{u} m_{F_{u}}\left\|\boldsymbol{T}^{(1)}(\mu)\right\| \psi e v_{l} \Lambda_{l} ; i_{\mathrm{D}_{l}} N_{l} F_{1_{l}} i_{\mathrm{H}_{l}} F_{l} m_{F_{l}}\right\rangle\right|^{2},
\end{aligned}
$$

where the reduced matrix element can be calculated:

$$
\begin{aligned}
& \left\langle\psi_{e v_{u}} \Lambda_{u} ; N_{u} i_{\mathrm{D}_{u}} F_{1_{u}} i_{\mathrm{H}_{u}} F_{u}\left\|\boldsymbol{T}^{(1)}(\mu)\right\| \psi_{e v_{l}} \Lambda_{l} ; N_{l} i_{\mathrm{D}_{l}} F_{1_{l}} i_{\mathrm{H}_{l}} F_{l}\right\rangle \\
= & (-1)^{F_{l}+i_{\mathrm{H}}+F_{1_{u}}+1} \sqrt{\left(2 F_{l}+1\right)\left(2 F_{u}+1\right)}\left\{\begin{array}{ccc}
i_{\mathrm{H}} & F_{l} & F_{1_{l}} \\
1 & F_{1_{u}} & F_{u}
\end{array}\right\} \\
\times & (-1)^{F_{1_{l}}+i_{\mathrm{D}}+N_{u}+1} \sqrt{\left(2 F_{1_{l}}+1\right)\left(2 F_{1_{u}}+1\right)}\left\{\begin{array}{ccc}
i_{\mathrm{D}} & F_{1_{l}} & N_{l} \\
1 & N_{u} & F_{1_{u}}
\end{array}\right\} \\
\times & \left\langle\psi_{e v_{u}} \Lambda_{u} N_{u}\left\|\boldsymbol{T}^{(1)}(\mu)\right\| \psi_{e v_{l}} \Lambda_{l} N_{l}\right\rangle .
\end{aligned}
$$

The following selection rules: $\Delta F=F_{u}-F_{l}=0, \pm 1(0 \leftrightarrow 0)$, and $\Delta i_{\mathrm{D}}=\Delta i_{\mathrm{H}}=0$ are deduced.

Finally, by using the finite rotation matrix $\mathcal{D}_{q^{\prime}}^{(2)}[\overline{81}$, the reduced matrix element in the molecule-fixed referential is deduced:

$$
\begin{aligned}
& \left\langle\psi_{e v_{u}} \Lambda_{u} N_{u}\left\|\boldsymbol{T}^{(1)}(\mu)\right\| \psi_{e v_{l}} \Lambda_{l} N_{l}\right\rangle \\
= & \left\langle\psi_{e v_{u}} \Lambda_{u} N_{u}\left\|\sum_{q^{\prime}} \mathcal{D}_{q^{\prime}}^{(2)^{*}} \boldsymbol{T}_{q^{\prime}}^{(1)}\left(\mu^{\prime}\right)\right\| \psi_{e v_{l}} \Lambda_{l} N_{l}\right\rangle \\
= & \sqrt{\left(2 N_{u}+1\right)\left(2 N_{l}+1\right)} \sum_{q^{\prime}}(-1)^{N_{u}-\Lambda_{u}}\left(\begin{array}{ccc}
N_{u} & 1 & N_{l} \\
-\Lambda_{u} & q^{\prime} & \Lambda_{l}
\end{array}\right)\left\langle\boldsymbol{T}_{-q^{\prime}}^{(1)}\left(\mu^{\prime}\right)\right\rangle,
\end{aligned}
$$

which provides the additional selection rules: $\Delta N=N_{u}-N_{l}=0, \pm 1$ and $\Delta \Lambda=\Delta_{u}-\Delta_{l}=0, \pm 1$.

When squaring Eq. B5], we recognize the usual Hönl-London factor

$$
\left(2 N_{u}+1\right)\left(2 N_{l}+1\right)\left(\begin{array}{ccc}
N_{u} & 1 & N_{l} \\
-\Lambda_{u} & \Delta \Lambda & \Lambda_{l}
\end{array}\right)^{2}
$$

For parallel transitions $\left[\Delta \Lambda=0,\left\langle\boldsymbol{T}_{-q}^{(1)}\left(\mu^{\prime}\right)\right\rangle=\left\langle\mu_{z^{\prime}}\right\rangle\right]$

$$
\begin{aligned}
& \left\langle\psi_{e v_{u}} \Lambda_{u} N_{u}\left\|\boldsymbol{T}^{(1)}(\mu)\right\| \psi_{e v_{l}} \Lambda_{l} N_{l}\right\rangle \\
= & (-1)^{N_{u}-\Lambda_{u}} \sqrt{\left(2 N_{u}+1\right)\left(2 N_{l}+1\right)}\left(\begin{array}{ccc}
N_{u} & 1 & N_{l} \\
-\Lambda & 0 & \Lambda
\end{array}\right)\left\langle\boldsymbol{T}_{0}^{(1)}\left(\mu^{\prime}\right)\right\rangle,
\end{aligned}
$$


where $\left\langle\boldsymbol{T}_{0}^{(1)}\left(\mu^{\prime}\right)\right\rangle$ is the molecular dipole moment (including the $N$ dependence) as defined in section III B.

It is worth pointing out that HITRAN ignores the individual hyperfine transitions [as compared with Eq. [26], but it deals with a degeneracy factor carrying out the nuclear spin statistic $g_{n s}$ which is obviously equal to $\left(2 i_{\mathrm{D}}+1\right) \times\left(2 i_{\mathrm{H}}+1\right)$. The existence of a sum rule on the $6 \mathrm{j}$ symbols allows us to check the sum intensity. 
[1] G. Herzberg. Rotation-vibration spectrum of the HD molecule. Nature, 166:563, 1950. doi:10.1038/166563a0.

[2] R.A. Durie and G. Herzberg. Forbidden transitions in diatomic molecules. V. The rotation-vibration spectrum of the hydrogendeuteride (HD) molecule. Can. J. Phys., 38:806-818, 1960. doi:10.1139/p60-086.

[3] L. Wolniewicz. Nonadiabatic energies of the ground state of the hydrogen molecule. J. Chem. Phys., 103:1792-1799, 1995. doi:10.1063/1.469753.

[4] L. Wolniewicz. Relativistic energies of the ground state of the hydrogen molecule. J. Chem. Phys., 99:1851-1868, 1993. doi:10.1063/1.465303.

[5] S. Bubin, F. Leonarski, M. Stanke, and L. Adamowicz. Charge asymmetry in pure vibrational states of the HD molecule. J. Chem. Phys., 130:124120-6, 2009. doi:10.1063/1.3094047.

[6] S. Bubin, M. Stanke, and L. Adamowicz. Complete pure vibrational spectrum of HD calculated without the Born-Oppenheimer approximation and including relativistic corrections. Phys. Rev. A, 83:042520-5, 2011. doi:10.1103/PhysRevA.83.042520.

[7] K. Strasburger. Modified adiabatic approximation: Charge asymmetry in $\mathrm{HD}^{+}$and HD. J. Chem. Phys., 131:134103-8, 2009. doi:10.1063/1.3241280.

[8] M. Stanke, S. Bubin, M. Molski, and L. Adamowicz. Non-Born-Oppenheimer calculations of the lowest vibrational energy of HD including relativistic corrections. Phys. Rev. A, 79:032507-8, 2009. doi:10.1103/PhysRevA.79.032507.

[9] L.M. Wang and Z.-C. Yan. Relativistic corrections to the ground state of $\mathrm{H}_{2}$ calculated without using the Born-Oppenheimer approximation. Phys. Rev. A, 97:060501-5, 2018. doi:10.1103/PhysRevA.97.060501.

[10] K. Pachucki and J. Komasa. Nonadiabatic rotational states of the hydrogen molecule. Phys. Chem. Chem. Phys., 20:247-255, 2018. doi:10.1039/c7cp06516g.

[11] N. Kirnosov, K. Sharkey, and L. Adamowicz. Charge asymmetry in the rovibrationally excited HD molecule. J. Chem. Phys., 140:104115-8, 2014. doi:10.1063/1.4867912.

[12] K. Pachucki and J. Komasa. Schrödinger equation solved for the hydrogen molecule with unprecedented accuracy. J. Chem. Phys., 144:164306-4, 2016. doi:10.1063/1.4948309.

[13] K. Pachucki and J. Komasa. Nonrelativistic energy levels of HD. Phys. Chem. Chem. Phys., 20:26297-26302, 2018. doi:10.1039/С8CР05493B.

[14] P. Czachorowski, M. Puchalski, J. Komasa, and K. Pachucki. Nonadiabatic relativistic correction in $\mathrm{H}_{2}$, $\mathrm{D}_{2}$, and HD. Phys. Rev. A, 98:052506-8, 2018. doi:10.1103/PhysRevA.98.052506.

[15] M. Puchalski, J. Komasa, and K. Pachucki. Nuclear spin-spin coupling in HD, HT, and DT. Phys. Rev. Lett., 120:083001-5, 2018. doi:10.1103/PhysRevLett.120.083001.

[16] F.M.J. Cozijn, P. Dupré, E.J. Salumbides, K.S.E. Eikema, and W. Ubachs. Sub-Doppler frequency metrology in HD for test of fundamental physics. Phys. Rev. Lett., 120:153002-5, 2018. doi:10.1103/PhysRevLett.120.153002.

[17] L.-G. Tao, A.-W. Liu, K. Pachucki, J. Komasa, Y.R. Sun, J. Wang, and S.-M. Hu. Toward a determination of the proton-electron mass ratio from the lamb-dip measurement of HD. Phys. Rev. Lett., 120:153001-5, 2018. doi:10.1103/PhysRevLett.120.153001.

[18] Th. Udem, R. Holzwarth, and T.W. Hänsch. Optical frequency metrology. Nature, 416:233-237, 2002. doi:10.1038/416233a.

[19] J. Komasa, K. Piszczatowski, G. Łach, M. Przybytek, B. Jeziorski, and K. Pachucki. Quantum electrodynamics effects in rovibrational spectra of molecular hydrogen. J. Chem. Theory Comput., 7:3105-3115, 2011. doi:10.1021/ct200438t.

[20] G. Buntkowsky, B. Walaszek, A. Adamczyk, Y. Xu, H.-H. Limbach, and B. Chaudret. Mechanism of nuclear spin initiated para-H2 
to ortho-H $\mathrm{H}_{2}$ conversion. Phys. Chem. Chem. Phys., 8:1929-1935, 2006. doi:10.1039/B601594H.

[21] P. Dupré. Saturated absorption and crossover resonances in a high-finesse cavity: Formalism and application to the hyperfine structure of jet-cooled $\mathrm{NO}_{2}$ by saturated-absorption cavity-ring-down spectroscopy. Phys. Rev. A, 85:042503-21, 2012. doi:10.1103/PhysRevA.85.042503.

[22] D. Bakalov, V.I. Korobov, and S. Schiller. High-precision calculation of the hyperfine structure of the HD ${ }^{+}$ion. Phys. Rev. Lett., 97:243001-4, 2006. doi:10.1103/PhysRevLett.97.243001.

[23] A.R.W. McKellar. Intensities of the dipole and quadrupole rotation-vibration spectra of HD. Can. J. Phys., 52:1144-1151, 1974. doi:10.1139/p74-152.

[24] L. Wolniewicz. On the computation of dipole transitions in the HD molecule. part II. Can. J. Phys., 54:672-679, 1976. doi:10.1139/p76-075.

[25] K. Pachucki and J. Komasa. Electric dipole rovibrational transitions in the HD molecule. Phys. Rev. A, 78:052503-7, 2008. doi:10.1103/PhysRevA.78.052503.

[26] S.M. Blinder. Dipole moment of HD. J. Chem. Phys., 32:105-110, 1960. doi:10.1063/1.1700880.

[27] W. Kołos and L. Wolniewicz. Vibronic energy and dipole moment of HD. J. Chem. Phys., 45:944-946, 1966. doi:10.1063/1.1727708.

[28] L. Wolniewicz and T. Kowalski. Rotational-vibrational dipole transitions in HD. Chem. Phys. Lett., 18:55-58, 1973. doi:10.1016/0009-2614(73)80337-9.

[29] P.R. Bunker. Forbidden transitions in homopolar isotopically unsymmetric diatomic molecules and the dipole moment of HD. J. Mol. Spectrosc., 46:119-129, 1973. doi:10.1016/0022-2852(73)90029-5.

[30] S.M. Blinder. Dipole moment of HD. II. J. Chem. Phys., 35:974-981, 1961. doi:10.1063/1.1701248.

[31] W.R. Thorson, J.H. Choi, and S.K. Knudson. Novel theory of the HD dipole moment. I. Theory. Phys. Rev. A, 31:22-33, 1985. doi:10.1103/PhysRevA.31.22.

[32] W.R. Thorson, J.H. Choi, and S.K. Knudson. Novel theory of the HD dipole moment. II. Computations. Phys. Rev. A, 31:34-42, 1985. doi:10.1103/PhysRevA.31.34.

[33] S.L. Hobson, E.F. Valeev, A.G. Császár, and J.F. Stanton. Is the adiabatic approximation sufficient to account for the post-BornOppenheimer effects on molecular electric dipole moments? Mol. Phys., 107:1153-1159, 2009. doi:10.1080/00268970902780262.

[34] M. Cafiero, S. Bubin, and L. Adamowicz. Non-Born-Oppenheimer calculations of atoms and molecules. Phys. Chem. Chem. Phys., 5:1491-1501, 2003. doi:10.1039/b211193d.

[35] M. Cafiero and L. Adamowicz. Non-Born-Oppenheimer isotope effects on the polarizabilities of $\mathrm{H}_{2}$. Phys. Rev. Lett., 89:073001-4, 2002. doi:10.1103/PhysRevLett.89.073001.

[36] D. Assafrāo and J.R Mohallem. The isotopic dipole moment of HDO. J. Phys. B: At. Mol. Opt., 40:F85-F91, 2007. doi:10.1088/09534075/40/5/F02.

[37] A. Birnbaum and J.D. Poll. Quadrupole transitions in the $\mathrm{H}_{2}$, $\mathrm{HD}$ and $\mathrm{D}_{2}$ molecules. J. Atmos. Sci., 26:943-945, 1969. doi:10.1175/1520-0469(1969)026<0943:QTITHH>2.0.CO;2.

[38] G.J. Rosasco, A.D. May, W.S. Hurst, L.B. Petway, and K.C. Smyth. Broadening and shifting of the Raman Q branch of HD. J. Chem. Phys., 90:2115-2124, 1989. doi:10.1063/1.456005.

[39] P. Wcisło, I.E. Gordon, H. Tran, Y. Tan, S.-M. Hu, A. Campargue, S. Kassi, D. Romanini, C. Hill, R.V. Kochanov, and L.S. Rothman. The implementation of non-Voigt line profiles in the HITRAN database: $\mathrm{H}_{2}$ case study. J. Quant. Spectrosc. Rad. Tr., 177:75-91, 2016. doi:10.1016/j.jqsrt.2016.01.024.

[40] R.L. Barger and J.H. Hall. Pressure shift and broadening of methane line at $3.39 \mu \mathrm{m}$ studied by laser-saturated molecular absorption. Phys. Rev. Lett., 22:4-8, 1969. doi:10.1103/PhysRevLett.22.4.

[41] S.N. Bagaev, E.V. Baklanov, and V.P. Chebotaev. Anomalous decrease of the shift of the center of the Lamb dip in low-pressure 
molecular gases. Sov. Phys. JETP Lett., 16:243-246, 1972.

[42] J.E. Thomas, M.J. Kelly, J-P. Monchalin, N.A. Kurnit, and A. Javan. Transit-time effects in power-broadened Doppler-free saturation resonances. Phys. Rev. A, 15:2356-2365, 1977. doi:10.1103/PhysRevA.15.2356.

[43] V.P. Chebotayev. Supernarrow saturated absorption resonances. Phys. Rep., 119:75-116, 1985.

[44] P. Dupré. Dipole saturated absorption modeling in frequency modulation spectroscopy: Dealing with a Gaussian beam, resonance narrowing. J. Quant. Spectrosc. Rad. Tr., 232:126-145, 2019. doi:10.1016/j.jqsrt.2019.04.015.

[45] R. Ciuryło and J. Szudy. Speed-dependent pressure broadening and shift in the soft collision approximation. J. Quant. Spectrosc. Rad. Tr., 57:411-423, 1997. doi:10.1016/S0022-4073(96)00078-7.

[46] N.F. Ramsey and H.R. Lewis. Theory of hydrogen deuteride in magnetic fields. Phys. Rev., 108:1246-1250, 1957. doi:10.1103/PhysRev.108.1246.

[47] W.E. Quinn, J.M. Baker, J.T. LaTourrette, and N.F. Ramsey. Radio-frequency spectra of hydrogen deuteride in strong magnetic fields. Phys. Rev., 112:1929-1940, 1958. doi:10.1103/PhysRev.112.1929.

[48] J.M. Brown and A. Carrington. Rotational Spectroscopy of Diatomic Molecules. Cambridge University Press, 2003.

[49] J. Gauss, Unpublished work.

[50] G.D. Purvis III and R.J. Bartlett. A full coupled-cluster singles and doubles model: The inclusion of disconnected triples. J. Chem. Phys., 76:1910-1918, 1982. doi:10.1063/1.443164.

[51] T.H. Dunning Jr. Gaussian basis sets for use in correlated molecular calculations. I. The atoms boron through neon and hydrogen. J. Chem. Phys., 90:1007-1023, 1989. doi:10.1063/1.456153.

[52] J. Gauss and J.F. Stanton. Analytic CCSD(T) second derivatives. Chem. Phys. Lett., 276:70-77, 1997. doi:10.1016/S00092614(97)88036-0.

[53] J.C. Light, I.P. Hamilton, and J.V. Lill. Generalized discrete variable approximation in quantum mechanics. J. Chem. Phys., 82:1400-1409, 1985. doi:10.1063/1.448462.

[54] J. Vázquez, M.E. Harding, J.F. Stanton, and J. Gauss. Vibrational energy levels via finite-basis calculations using a quasi-analytic form of the kinetic energy. J. Chem. Theory Comput., 7:1428-1442, 2011. doi:10.1021/ct100711u.

[55] J.F. Stanton, J. Gauss, L. Cheng, M.E. Harding, D.A. Matthews, and P.G. Szalay. CFOUR, coupled-cluster techniques for computational chemistry, a quantum-chemical program package. http://www.cfour.de. URL: http://www.cfour.de

[56] H. Lefebvre-Brion and R.W. Field. The Spectra and Dynamic of Diatomic Molecules. Academic Press, 2004.

[57] G.R. Gunther-Mohr, C.H. Townes, and J.H. Van Vleck. Hyperfine structure in the spectrum of $\mathrm{N}^{14} \mathrm{H}_{3}$. II. Theoretical discussion. Phys. Rev., 94:1191-1203, 1954. doi:10.1103/PhysRev.94.1191.

[58] W.H. Flygare. Spin-rotation interaction and magnetic shielding in molecules. J. Chem. Phys., 41:793-800, 1964. doi:10.1063/1.1725962.

[59] M.B. Kellogg, I.I. Rabi, N.F. Ramsey, Jr., and J. R. Zacharias. The magnetic moments of the proton and the deuteron. The radiofrequency spectrum of $\mathrm{H}_{2}$ in various magnetic fields. Phys. Rev., 56:728-743, 1939. doi:10.1103/PhysRev.56.728.

[60] G. Breit. The effect of retardation on the interaction of two electrons. Phys. Rev., 34:553-573, 1929. doi:10.1103/PhysRev.34.553.

[61] Renny Terry. Quantities, Units and Symbols in Physical Chemistry. The Royal Society of Chemistry, 3rd edition, 2007. doi:10.1039/9781847557889.

[62] C.H. Townes and A.L. Schawlow. Microwave Spectroscopy. Dover, 1975.

[63] I.C. Bowater, J.M. Brown, and A. Carrington. Microwave spectroscopy of nonlinear free radicals I. General theory and application to the Zeeman effect in HCO. Proc. R. Soc. Lond. A, 333:265-288, 1973. doi:10.1098/rspa.1973.0062.

[64] U. Fano and G. Racah. Irreducible Tensorial Sets. Academic Press, New York, 1959.

[65] A. Messiah. Quantum Mechanics. Dover, 1999. 
[66] G. Herzberg. Molecular Spectra and Molecular Structure, I Spectra of Diatomic Molecules. Krieger Publishing Company, Malabar, FL, second edition, 1989.

[67] R.F. Code and N.F. Ramsey. Molecular-beam magnetic resonance studies of HD and $\mathrm{D}_{2}$. Phys. Rev. A, 4:1945-1959, 1971. doi:10.1103/PhysRevA.4.1945.

[68] M. Pavanello, W.-C. Tung, and L. Adamowicz. Determination of deuteron quadrupole moment from calculations of the electric field gradient in $\mathrm{D}_{2}$ and HD. Phys. Rev. A, 81:042526-6, 2010. doi:10.1103/PhysRevA.81.042526.

[69] H.Y. Carr and E.M. Purcell. Interaction between nuclear spins in HD gas. Phys. Rev., 88:415-416, 1952. doi:10.1103/PhysRev.88.415.2.

[70] N.F. Ramsey and E.M. Purcell. Interactions between nuclear spins in molecules. Phys. Rev., 85:143-144, 1952. doi:10.1103/PhysRev.85.143.

[71] L. Ulivi, P. de Natale, and M. Inguscio. Pure rotational spectrum of hydrogen deuteride by far-infrared fourier transform spectroscopy. Astrophys. J., 378:L29-L31, 1991. doi:10.1086/186134.

[72] Perl data language, scientific computing with perl. http://pdl.perl.org.

[73] S. Kassi and A. Campargue. Electric quadrupole and dipole transitions of the first overtone band HD by CRDS between 1.45 and $1.33 \mu \mathrm{m}$. J. Mol. Spectrosc., 267:36-42, 2011. doi:10.1016/j.jms.2011.02.001.

[74] R. Herman and R.F. Wallis. Influence of vibration-rotation interaction on line intensities in vibration-rotation bands of diatomic molecules. J. Chem. Phys., 23:637-647, 1955. doi:10.1063/1.1742069.

[75] P.R. Bunker. Molecular Symmetry and Spectroscopy. Academic Press, 1979.

[76] G.B. Field, W.B. Somerville, and K. Dressler. Hydrogen molecules in astronomy. Annu. Rev. Astron. Astrophys., 4:207-243, 1966. doi:10.1146/annurev.aa.04.090166.001231.

[77] E. Fasci, A. Castrillo, H. Dinesan, S. Gravina, L. Moretti, and L. Gianfrani. Precision spectroscopy of HD at 1.38 $\mu \mathrm{m}$. Phys. Rev. A, 98:022516-6, 2018. doi:10.1103/PhysRevA.98.022516.

[78] P. Dupré. Sub-Doppler noise-immune cavity-enhanced optical heterodyne molecular spectrometry modeling: from Doppler broadening to cross-sideband resonances. J. Opt. Soc. Am. B, 32:838-860, 2015. doi:10.1364/JOSAB.32.000838.

[79] R.L. Cook and F.C. De Lucia. Application of the theory of irreducible tensor operators to molecular hyperfine structure. Am. J. Phys., 39:1433-1454, 1971. doi:10.1119/1.1976693.

[80] R.N. Zare. Angular Momentum. John Wiley \& Sons, 1988.

[81] A.R. Edmonds. Angular Momentum in Quantum Mechanics. Princeton University Press, New Jersey, third edition, 1974. 